J3eA - Vol. 2 - 7 (2003).

DOI : 10.1051/bib-j3ea:2003007

\title{
Une introduction aux télécommunications optiques par la simulation de systèmes simples
}

\author{
E. Cassan * (IEF, Université Paris-Sud Orsay)
}

Mis en ligne le 4 juin 2003.

\begin{abstract}
Résumé
Cet article présente une expérience menée à l'université Paris-Sud pour l'enseignement des télécommunications optiques au sein de la maîtrise EEA et du DESS Systèmes Électroniques d'Orsay. Un logiciel commercial (COMSIS), permettant une description fonctionnelle des chaînes de communication par fibre optique, est utilisé au cours de séances de TP sur cette thématique. Cette approche permet une étude globale des liaisons simples ou mutiplexées en longueur d'onde (WDM), en mettant l'accent sur les principales limitations introduites par les différents composants optiques (fibre, source laser, amplificateur optique, etc.). Chaque élément particulier est décrit par un ensemble de propriétés à fournir au logiciel et dont la connaissance nécessite, de la part des étudiants, une bonne assimilation du cours. L'utilisation du logiciel permet donc à ce titre d'insister sur des notions mal maîtrisées par les étudiants.
\end{abstract}

Mots-clés : télécommunications, fibre optique, diode laser, photodétecteur, amplificateur EDFA, facteur Q, taux d'erreur par bit.

(C) EDP Sciences, 2003.

\begin{abstract}
Niveau de connaissances requis. Notions sur la physique des SC et des composants, notions sur les modulations analogiques et numériques, cours sur les télécommunications par fibre optique.

Niveau des étudiants. Maîtrise EEA.

* Eric Cassan ${ }^{1}$, ancien élève de l'ENS de Cachan, a été reçu à l'agrégation de physique appliquée en 1995 et a obtenu le doctorat en sciences de l'université Paris-Sud en janvier 2000. Maître de conférences depuis septembre 2000, il enseigne dans les domaines des télécommunications optiques, de l'optique intégrée, de la physique des composants, et de l'électronique analogique, au niveau de la maîtrise EEA, des DESS Systèmes Electroniques et Réseaux et télécoms d'Orsay, du magistère en EEA (Université d'Orsay/ENS Cachan), et de la licence EEA d'Orsay.
\end{abstract}

Ses thèmes de recherche, développés à l'Institut d'Électronique Fondamentale d'Orsay (UMR CNRS 8622), sont centrés sur l'étude et la caractérisation de composants actifs (modulateurs de lumière, photodétecteurs) et passifs (microguides d'ondes, virages, diviseurs de faisceaux) d'optoélectronique intégrée dans la filière silicium. Les deux principaux champs d'application de ces thèmes de recherche concernent d'une part l'introduction d'interconnexions optiques au sein des circuits intégrés microélectroniques CMOS, et d'autre part la mise au point de composants silicium bas coût pour les télécommunications optiques.

a e-mail : eric.cassan@ief.u-psud.fr

\footnotetext{
${ }^{1}$ Université Paris-Sud Orsay, Institut d'Électronique Fondamentale, UMR CNRS 8622, Bâtiment 220, F-91405 Orsay Cedex, France.
} 


\section{Contexte et objectifs}

Les télécommunications par fibre optique, dont le développement a sensiblement débuté il y a vingt-cinq ans (voir l'historique des communications par fibre optique en annexe A), connaissent un certain essor depuis l'introduction, il y a cinq ans environ, du multiplexage en longueur d'onde (en anglais Wavelength Division Multiplexing, ou WDM). Cette technologie a pour point fort d'exploiter avantageusement l'immense bande passante des fibres optiques. Elle permet actuellement de transmettre 160 canaux en longueurs d'onde, mutliplexés chacun dans le temps à un débit binaire de 40 Gbits.s ${ }^{-1}$, soit un débit global de 6,4 Tbits. $\mathrm{s}^{-1}$, ou encore 100 millions de communications téléphoniques à 64 kbits sur une unique fibre optique.

Les systèmes de télécommunication optiques mettent en jeu un ensemble d'éléments, à commencer par les composants d'émission/réception de la lumière, le support de propagation qu'est la fibre optique, les amplificateurs optiques, ainsi que les composants spécifiques pour le WDM. À ces fonctions purement optiques et/ou optoélectroniques s'ajoutent des circuits électroniques rapides permettant de moduler et d'amplifier les signaux émis et recueillis, de récupérer les signaux de référence, ainsi que des organes de haut niveau de gestion du réseau.

Cet article a pour but de présenter l'utilisation qui peut être faite de la simulation de systèmes simples pour une introduction aux télécommunications par fibre optique. Il s'appuie sur une expérience menée cette année au sein du DESS Systèmes Électroniques d'Orsay sous la forme de TP utilisant le logiciel commercial COMSIS [1]. Cet enseignement a été également introduit cette année dans le programme maîtrise EEA d'Orsay sous la forme d'un TD-TP. Les exercices proposés ont un but très pédagogique, dans le sens où ils permettent de montrer l'impact de tel ou tel phénomène sur les performances du système, même s'ils ne sont pas réalistes d'un point de vue du réseau de télécommunications optiques. La partie 2 de cet article résume quelques-unes des techniques et des propriétés des liaisons par fibre optique, utilisées dans la partie 3, dont l'objectif est de détailler deux exemples d'étude menées à Orsay.

\section{Télécommunications par fibre optique}

Les télécommunications optiques constituent un domaine multidisciplinaire. Elles mettent en jeu des composants et circuits d'émission et de réception, un support de transmission aux propriétés bien particulières et des techniques appropriées de codage, de modulation et de récupération des informations. D'un point de vue pédagogique, il est nécessaire d'aborder auprès des étudiants l'ensemble de ces aspects pour une bonne compréhension des systèmes mis en jeu.

\subsection{Liaisons point à point mono-longueur d'onde}

De la même manière que dans le domaine des transmissions radio, la transmission d'informations entre deux points par une fibre optique peut être effectuée selon une modulation, qui est soit analogique (d'amplitude, de fréquence, ou de phase), soit numérique. Dans la très grande majorité des cas, la modulation est de type numérique, par variation directe du courant d'une diode laser ou utilisation d'un modulateur externe. La figure 1 représente une liaison optique numérique sous test à un débit binaire $\mathrm{D}=10 \mathrm{Gbits} . \mathrm{s}^{-1}$, point à point et monolongueur d'onde $(\lambda=1,55 \mu \mathrm{m})$. L'utilisation de cette longueur d'onde est liée aux propriétés de la fibre optique monomode en silice. 


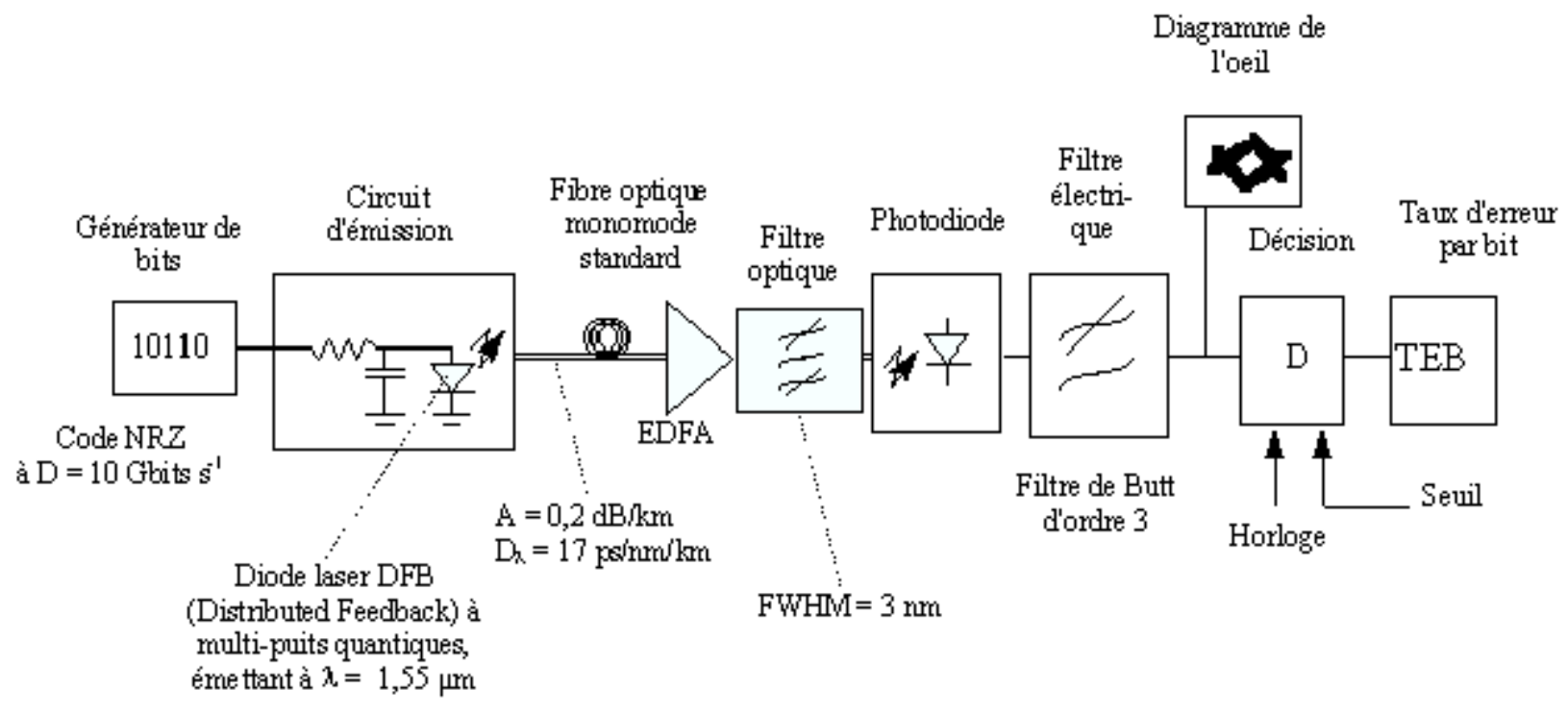

Fig. 1. Exemple de transmission numérique par fibre optique.

Le principe de la liaison est le suivant :

- L'information est codée en amont. Sur la figure 1, ce codage est illustré par l'utilisation d'un générateur de bits pseudo-aléatoire.

- Celui-ci attaque un circuit de modulation rapide, qui module le courant de la diode laser performante (diode laser à cavité de Bragg distribuée : en anglais Distributed Feedback ou DFB).

- Le signal optique est introduit dans une fibre monomode, puis amplifié optiquement par le biais d'un amplificateur à fibre dopée Erbium (en anglais Erbium Doped Fiber Amplifier ou EDFA), afin d'améliorer le rapport signal à bruit après photodétection.

- Après détection au moyen d'une photodiode, la qualité globale de la liaison numérique peut être évaluée grâce à un diagramme de l'oeil, à partir duquel le meilleur moment d'échantillonnage (Horloge) et le niveau seuil de tension (Seuil) pour la prise de décision sont choisis.

Cette courte description montre que même une liaison aussi simple qu'une liaison point à point mono-longueur d'onde comporte déjà un assez grand nombre d'éléments et de facteurs à prendre en considération. Les limitations d'une telle liaison peuvent être liées aux limites physiques des diodes laser modulées en courant, aux caractéristiques de dispersion et d'atténuation des fibres optiques, aux caractéristiques d'amplification, de saturation et de bruit de l'amplificateur optique, ainsi qu'à la sensibilité et rapidité du circuit de réception.

Pour la réalisation de communications fiables, il est nécessaire que l'ensemble des composants soient compatibles, et que leurs caractéristiques individuelles améliorent, plutôt que ne dégradent, les caractéristiques du système global. Un outil de simulation de la liaison, prenant en compte l'essentiel des caractéristiques physiques de chacun des éléments, peut alors s'avérer intéressant. Ce type d'analyse permet en effet de séparer les effets intervenant dans le fonctionnement global de la chaîne de communication et de dimensionner une liaison en fonction d'un cahier des charges, portant par exemple sur la longueur totale de la liaison et sur un niveau requis de taux d'erreur par bit $\left(\mathrm{ex}: \mathrm{TEB}=10^{-12}\right.$ ). Ce type d'étude nécessite de la part des étudiants une double culture : une bonne connaissance, d'une part des caractéristiques physiques des composants mis en jeu ainsi que de leurs limitations, et d'autre part de leurs principales caractéristiques fonctionnelles.

\subsection{Composants d'émission/réception}

Le principe de fonctionnement des composants d'émission/réception repose sur l'interaction lumière/matière dans les semiconducteurs III/V (émission spontanée, émission stimulée, et absorption), ainsi que sur la physique des dispositifs électroniques, la jonction PN en particulier [2-6]. 


\section{a) Composants d'émission}

On distingue classiquement les diodes électroluminescentes (DEL) et les diodes laser (DL) selon le type de mécanisme mis en jeu pour l'émission de lumière (émission spontanée pour les DEL et émission stimulée pour les DL). Dans le cas des liaisons haut débit, seules les diodes laser, nettement plus performantes, sont utilisées.

Dans une description de type « système » des liaisons par fibre optique, les diodes laser interviennent essentiellement par leurs caractéristiques statiques (annexe B) et par leurs caractéristiques dynamiques (annexe C).

\section{b) Composants de réception}

La détection est un point clef des liaisons par fibre optique. En général, les signaux reçus sont très faibles (typiquement le $\mathrm{nW}$ ) et rapides (le débit binaire des liaisons peut atteindre $40 \mathrm{Gbits} . \mathrm{s}^{-1}$ ). Il est donc nécessaire que le système de détection soit sensible et rapide. Le rayonnement incident crée, par génération bande à bande, des paires électron-trou séparées par un champ électrostatique, puis collectées par des contacts électriques. Ce flux de charges donne lieu à un photocourant $I_{p h}$. Le système de détection comprend, en plus de l'élément photodétecteur proprement dit (par exemple une photodiode PIN), un ensemble de circuits électroniques, à commencer par un pré-amplificateur faible bruit.

Du point de vue des liaisons par fibre, les principaux paramètres qui interviennent pour caractériser la détection sont : la longueur d'onde de coupure, la sensibilité du détecteur $S_{\lambda}(\mathrm{A} / \mathrm{W})$ qui chiffre le rapport entre le photocourant recueilli et la puissance optique incidente, le rendement quantique, et le courant d'obscurité du détecteur (voir les caractéristiques des photodétecteurs en annexe D).

\subsection{Fibre optique}

La fibre optique est le support de propagation de la lumière. Il s'agit d'un guide d'onde diélectrique à géométrie cylindrique. On distingue principalement les fibres optiques multimodes et les fibres optiques monomodes, selon le nombre de modes pouvant se propager compte tenu de la géométrie de la fibre (essentiellement son diamètre de coeur) et le profil de dopage coeur/gaine (Fig. E-2 en annexe E).

Du point de vue de la propagation de la lumière, les propriétés principales d'un fibre sont d'une part son atténuation (pertes $\alpha$ en $\mathrm{dB} / \mathrm{km}$ ) (Fig E-3 en annexe E), et d'autre part ses propriétés de dispersion.

La principale source de dispersion dans les fibres multimodes est la dispersion intermodale, liée à une différence de vitesse de propagation des différents modes présents (Fig. E-4 en annexe E). La dispersion se traduit par un étalement temporel des impulsions lumineuses se propageant dans la fibre, qui peut conduire à un recouvrement total des impulsions codant les différents bits d'information.

Pour les fibres monomodes, utilisées pour l'établissement de liaisons rapides, la dispersion chromatique intervient seule. Elle est liée au caractère non-monochromatique de la source de lumière et au fait que la vitesse de propagation du mode de la fibre varie avec la longueur d'onde. L'étalement temporel des impulsions s'exprime par la relation suivante, où $D_{\lambda}$ est le coefficient de dispersion de la fibre en $\mathrm{ps} /(\mathrm{nm} . \mathrm{km}), \Delta \lambda$ la largeur spectrale effective de la diode laser, et $L$ la longueur de la liaison en $\mathrm{km}$ :

$$
\Delta T=D_{x} \times \Delta \lambda \times L
$$

Par exemple, avec : $D_{\lambda}=17 \mathrm{ps} \cdot \mathrm{km}^{-1} \cdot \mathrm{nm}^{-1}, \Delta \lambda=0,5 \mathrm{~nm}, L=10 \mathrm{~km}$, l'étalement typique des impulsions est de $\Delta T=85$ ps. Le débit binaire maximum est de l'ordre de $1 / 2 \Delta T$, soit 5,9 GHz (Fig. E-5 en annexe E). 


\subsection{Amplificateurs optiques}

Les amplificateurs optiques sont destinés à amplifier les signaux optiques sans recourir à des solutions optoélectroniques basées sur des conversions de type optique/électronique et électronique/optique (photodétection, amplification électronique, puis émission laser à la même longueur d'onde à partir du signal amplifié électroniquement). Parmi les amplificateurs optiques, figurent principalement les amplificateurs à fibre dopée Erbium (EDFA, de l'acronyme anglais Erbium Doped Fiber Amplifier), les amplificateurs à semiconducteurs et les amplificateurs Raman [7,8]. Leur principe repose toujours sur la possibilité d'amplification optique par émission stimulée de lumière, de la même manière que pour les lasers. Nous ne présentons ici que l'amplificateur EDFA, qui occupe une place importante dans les liaisons par fibre, notamment en tant qu'amplificateur de ligne.

Un amplificateur à fibre dopée Erbium consiste en une courte section de fibre optique (typiquement quelques dizaines de mètres), possédant une fraction de l'élément terre rare Erbium sous la forme d'ions $\mathrm{Er}^{3+}$. L'amplification optique repose sur la possibilité d'amplification stimulée du signal. Les ions $\mathrm{Er}^{3+}$, insérés dans la matrice amorphe de silice, matériau constituant la fibre optique, présentent un diagramme énergétique simplifié à trois niveaux (Fig. F-1 en annexe F).

Le principe de fonctionnement de l'EDFA est le suivant (Fig. F-2 en annexe) :

- Le signal à amplifier est mélangé à un signal de pompe de forte puissance (de 10 à $200 \mathrm{~mW}$ typiquement) grâce à un multiplexeur de longueurs d'onde, et le signal résultant est injecté dans la fibre dopée $\mathrm{Er}^{3+}$. Deux longueurs d'ondes sont disponibles pour le signal de pompe : $980 \mathrm{~nm}$ ou $1480 \mathrm{~nm}$.

- Le signal de pompe provoque une inversion de population des ions $\mathrm{Er}^{3+}$ et le milieu devient amplificateur vis-à-vis du signal optique d'entrée.

- Lorsque l'émission stimulée domine, elle donne lieu à de l'amplification optique. Cela dit, trois phénomènes coexistent toujours : absorption, émission spontanée et émission stimulée.

Quantitativement, les caractéristiques techniques principales des EDFA sont les suivantes :

- le gain de l'amplificateur (rapport en décibels $(\mathrm{dB})$ de la puissance de sortie sur la puissance en entrée) : typiquement $20 \mathrm{~dB}$;

- $\quad$ le niveau de puissance de saturation $P_{S}$ du gain de l'amplificateur, qui chiffre la puissance de sortie pour laquelle le gain de l'amplificateur sature déjà : typiquement 1 à $10 \mathrm{~mW}$;

- la bande spectrale d'amplification $\Delta \lambda$ : typiquement $25 \mathrm{~nm}$;

- $\quad$ le facteur de bruit $F$, qui chiffre la dégradation du rapport signal à bruit à la traversée de l'amplificateur du fait de l'amplification de l'émission spontanée (ASE) : typiquement 6 à 8 dB.

Les applications se situent au coeur des domaines des télécommunications numériques ou analogiques : amplificateur de puissance (linéarité, puissance de sortie), amplificateur en ligne (linéarité, puissance de sortie, bruit), préamplificateur (bruit).

\subsection{Multiplexage des signaux optiques}

La fréquence de modulation maximale lors d'une modulation analogique, ou le débit binaire maximal lors d'une modulation numérique, constituent en général une fraction de la fréquence porteuse. À ce titre, la porteuse optique présente l'avantage d'être extrêmement élevée : aux longueurs d'ondes des télécommunications $(\lambda=0,85 \mu \mathrm{m}, \lambda=1,31 \mu \mathrm{m}$ et surtout $\lambda=1,55 \mu \mathrm{m})$, la porteuse optique a une fréquence de l'ordre de quelques $10^{14} \mathrm{~Hz}$. Sur le principe, il est donc envisageable de transmettre des débits de données jusqu'à 1000 Gbits.s ${ }^{-1}$. Les circuits électroniques actuels (notamment de modulation des lasers ou modulateurs externes, ceux nécessaires au niveau de la réception) permettent actuellement un débit de $40 \mathrm{Gbits}^{-1} \mathrm{~s}^{-1}$. Lorsque de tels débits ne sont pas nécessaires, par exemple pour la transmission de données téléphoniques à $64 \mathrm{kbits} . \mathrm{s}^{-1}$, il est possible de profiter de ces possibilités en exploitant la technique du multiplexage temporel (TDM pour l'acronyme anglais Time Division Multiplexing) (annexe G). Un débit de $40 \mathrm{Gbits.s}^{-1}$ correspond ainsi à approximativement 600000 conversations téléphoniques véhiculées par la même fibre véhiculant une unique longueur d'onde.

L'optique permet une autre forme de multiplexage, le multiplexage en longueur d'onde ou WDM (Wavelength Division Multiplexing). Partant du constat que dans le domaine des faibles puissances optiques, la propagation de la lumière est régie par des lois linéaires (équations de Maxwell dans lesquelles on néglige les termes nonlinéaires liés à la constitution de la fibre optique), il est apparu possible de faire se propager sur la même fibre 
non pas une longueur d'onde mais plusieurs. Ces signaux, à des longueurs d'onde différentes, peuvent être cadencés à des débits binaires différents ou peuvent correspondre à des formats de codage différents, sans que cela ne pose aucun problème, dans la mesure où les signaux n'interagissent pas entre eux (ce qui n'est jamais tout à fait vrai) [7].

En première approximation, le débit binaire global est ainsi multiplié par le nombre de canaux en longueurs d'onde (typiquement jusqu'à 160). Cette technique permet de dépasser les $1000 \mathrm{Gbits}^{\mathrm{s}} \mathrm{s}^{-1}$ véhiculé par une unique fibre optique. De nombreux composants passifs d'optique intégrée ont été conçus pour rendre viable les communications par fibre basées sur le WDM : multiplexeurs/démultiplexeurs en longueur d'onde, filtres optiques, convertisseurs de longueurs d'onde, etc. [9,10,11] (annexe G).

\section{Apport de la simulation de systèmes simples}

\subsection{Outil de simulation}

Parmi les logiciels dédiés aux communications numériques, peu offrent la possibilité de décrire des liaisons optiques. Notre choix s'est porté sur le logiciel COMSIS, dans sa version incluant une bibliothèque de composants optiques. Le coût de ce produit est de l'ordre de 10000 euros TTC pour une licence site.

\section{2. Étude d'une liaison point à point mono-longueur d'onde $(\lambda=1,55 \mu \mathrm{m})$}

La figure 2 présente le schéma de principe d'une liaison numérique point à point mono-longueur d'onde $(\lambda=1,55 \mu \mathrm{m})$ étudiée par les étudiants. Elle présente une diode laser pour l'émission de la lumière (Fig. H-1, annexe H.1.), dont le courant est modulé suivant un flux de bits déterminé par le générateur de bits placé en amont (Fig. H1-1, annexe H1.1.), suivie de deux tronçons de fibre optique monomode (Fig. H1-1, annexe H.1.), séparés par un amplificateur à fibre dopée Erbium utilisé en amplificateur optique de ligne (Fig. H1-1, annexe H.1.). La liaison est terminée par une photodiode PIN (Fig. H1-1, annexe H.1.) pour la récupération sous forme électrique des informations. Chaque élément de la liaison possède une description que l'on demande aux étudiants d'analyser et de paramétrer (annexe H.1.). 


\section{Grandeurs mesurables}

\section{Description à fournir}

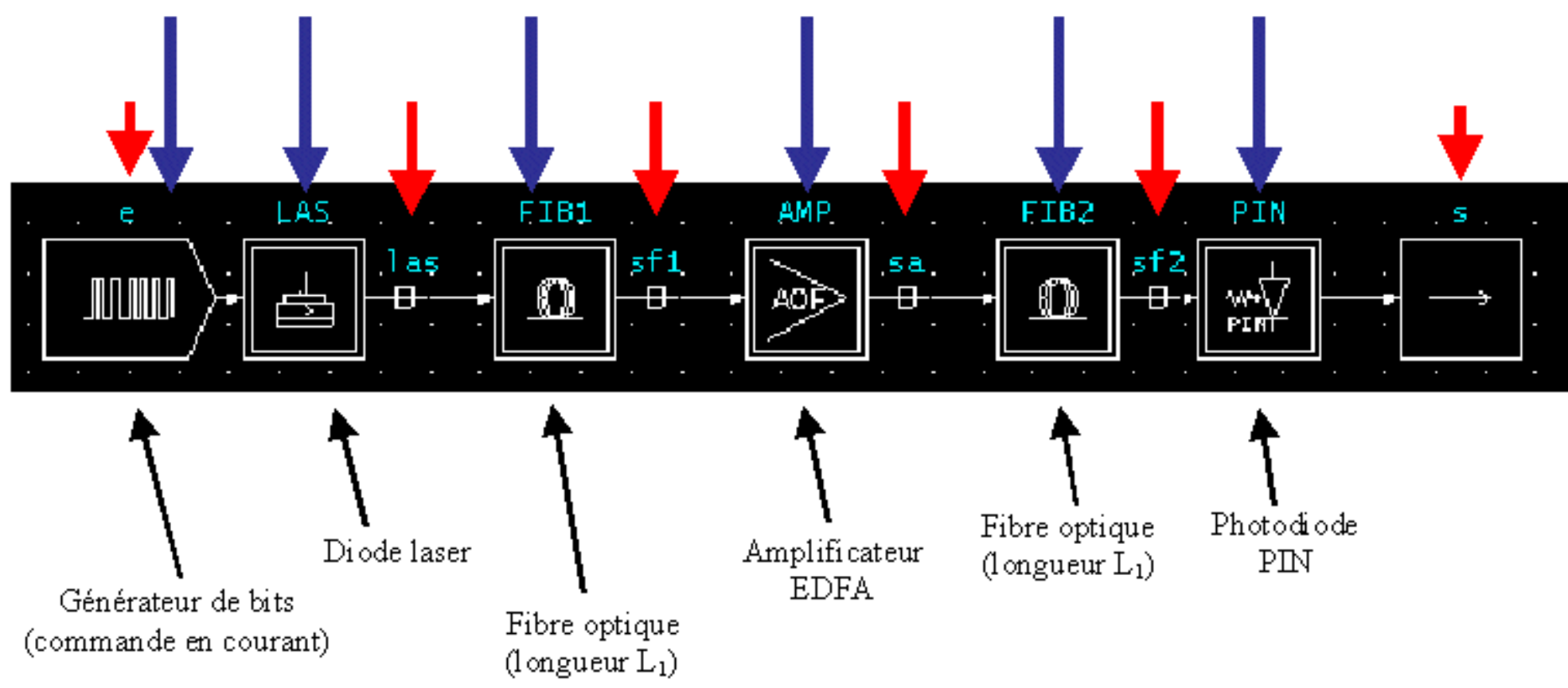

Fig. 2. Synoptique de la liaison point à point mono-longueur d'onde étudiée à l'aide du logiciel COMSIS. Source : Copie d'écran du logiciel COMSIS [1]

Pratiquement tous les paramètres introduits interviennent dans l'évaluation des performances de ce type de liaison :

- Le débit binaire et les courants minimum et maximum délivrés par le générateur de bits. Ces derniers vont fixer, relativement à la caractéristique statique de la diode laser les niveaux de puissance optique codant le niveau logique « 0 » et le niveau logique « 1 ».

- La longueur d'onde centrale de la diode laser (environ 1,55 $\mu \mathrm{m}$ pour les applications haut débit), les caractéristiques statiques du composant d'émission (courant de seuil, rendement puissance/courant de la caractéristique $P_{\text {opt }}=P_{\text {opt }}(I)$ ), ainsi que les caractéristiques dynamiques de la diode (taux d'amortissement, chirp, etc.).

- Les pertes et la dispersion chromatique de la fibre optique monomode, introduites par l'intermédiaire des coefficients de pertes en $\mathrm{dB} / \mathrm{km}$ et de dispersion en $\mathrm{ps} /(\mathrm{nm} \times \mathrm{km})$.

- Le gain de l'EDFA en $\mathrm{dB}$ et son facteur de bruit (qui fixe la dégradation du rapport signal à bruit en raison de l'amplification de l'émission spontanée).

- La sensibilité de la photodiode PIN (A/W) et son courant d'obscurité

Ces paramètres peuvent être introduits sous forme de variables et donc modifiés à tout moment pour voir l'effet de tel ou tel phénomène sur les performances globales de la liaison. Il est également possible de passer d'une description « système » des différents éléments à une description « physique ». Dans le premier cas, par exemple, le gain de l'EDFA est stipulé explicitement, alors que dans le second, on précise la concentration en ions erbium $\mathrm{Er}^{3+}$ dans la matrice de silice, la longueur de la section de fibre dopée, etc. Deux types d'analyse de la liaison sont principalement possibles : l'« analyse statique », grâce à laquelle il est possible d'analyser les principales caractéristiques de chaque composant, et l'« analyse temporelle », grâce à laquelle on peut visualiser et effectuer des traitements sur l'ensemble des grandeurs de la liaison complète. 


\title{
a) Analyse statique
}

\author{
Exp1 (annexe H.2.)
}

Dans un premier temps, on demande aux étudiants de visualiser et d'analyser les caractéristiques des différents éléments de la liaison grâce à l'outil d'analyse statique. Cette étape préliminaire, avant la simulation de la liaison complète, permet de s'assurer que les principales notions relatives aux différentes fonctions optiques mises en jeu (émission, propagation, amplification, détection) sont maîtrisées par les étudiants, ainsi que le fonctionnement des composants associés (diode laser, fibre optique, amplificateur EDFA, photodiode). À titre d'exemple, nous traitons ici du cas de l'amplificateur EDFA, dont les caractéristiques fonctionnelles relient principalement la puissance de pompe $P_{P}$ et la puissance du signal à amplifier $P_{i n}$ au gain $G$ de l'amplificateur (EXP 1 en annexe H.2.).

\section{b) Analyse temporelle}

L'étude du système complet commence avec l'analyse temporelle. Les étudiants sont invités à se servir d'une configuration initiale des valeurs de tous les paramètres introduits, comme point de référence, et à faire varier successivement les valeurs de plusieurs d'entre eux afin d'en observer les conséquences sur la qualité de la liaison.

Afin d'essayer de découpler les différents phénomènes mis en jeu, il est demandé aux étudiants d'observer les signaux de la liaison de proche en proche, en partant de la source et en allant jusqu'à la détection.

\section{Exp2 : Forme des impulsions de la diode laser (annexe H.3.)}

Pour des caractéristiques données du générateur de bits (excepté le débit binaire) et de la diode laser (voir sur la figure H1-1 les paramètres du générateur et de la diode laser, annexe H.1.), on étudie l'effet du débit des données sur la forme des impulsions optiques délivrées par la diode laser. Aucune information ne pourra en effet être interprétée en bout de liaison si la diode laser fonctionne d'ores et déjà hors de son domaine «normal» de fonctionnement. La diode laser considérée peut être modulée en courant à un débit binaire de l'ordre de 1 Gbits.s , au maximum 5 Gbits.s ${ }^{-1}$ (EXP 2, annexe H.3.).

Exp3 : Effet de la dispersion chromatique de la fibre (annexe H.4.)

Pour les deux débits binaires de 1 Gbits.s $\mathrm{s}^{-1}$ et $5 \mathrm{Gbits}^{-1} \mathrm{~s}^{-1}$, on demande aux étudiants d'étudier l'effet de la dispersion chromatique seule de la fibre monomode sur les signaux de la liaison. Pour cela, il suffit de spécifier un coefficient de pertes nul. La valeur retenue du coefficient de dispersion est quant à elle de $17 \mathrm{ps} /(\mathrm{nm} . \mathrm{km})$ (valeur typique pour une fibre monomode standard à la longueur d'onde de 1,55 $\mu \mathrm{m}$ ). La longueur $L_{l}$ de la première section de fibre est ici un paramètre que l'on fait varier, et l'on observe simultanément la puissance des impulsions laser et la puissance optique en sortie de la première section de fibre (EXP 3, annexe H.4.). L'analyse comparative de ces résultats confirme que la dispersion chromatique est d'autant plus forte que le débit binaire est élevé et que la longueur de la liaison est importante. L'apport de la simulation est de permettre une visualisation des déformations introduites par la dispersion chromatique, et d'évaluer quantitativement les limites en débit binaire et en longueur de liaison. Dans le cas présent, la dispersion chromatique est peu gênante à 1 Gbits. $\mathrm{s}^{-1}$. En revanche, son effet devient particulièrement pénalisant à 5 Gbits. $\mathrm{s}^{-1}$, même pour une liaison relativement courte $(200 \mathrm{~km})$.

\section{Exp4 : Conditions d'utilisation de l'EDFA (annexe H.5.)}

On demande ensuite aux étudiants d'étudier les conditions d'utilisation de l'amplificateur optique EDFA. Pour cela, on se fixe un débit binaire de $1 \mathrm{Gbits}^{-1}{ }^{-1}$, et tenant compte des pertes $(\alpha=0,2 \mathrm{~dB} / \mathrm{km})$ et de la dispersion chromatique $\left(D_{\lambda}=17 \mathrm{ps} / \mathrm{nm} / \mathrm{km}\right)$ de la fibre optique monomode, on évalue l'effet de la longueur $L_{l}$ de la première section de fibre en observant les signaux sfl et sa, respectivement en entrée et en sortie de l'amplificateur EDFA (EXP 4, annexe H.5.).

L'analyse comparative des résultats obtenus montre qu'il faut utiliser l'EDFA avec un niveau de signal suffisamment faible pour que le gain de l'amplificateur ne sature pas (si le niveau du signal n'est pas faible, il n'y a d'ailleurs pas intérêt à amplifier !), mais pas trop pour ne pas être pénalisé par le bruit d'amplification de l'émission spontanée (ASE). Les ordres de grandeurs correspondant peuvent en fait être directement évalués à partir des caractéristiques données par l'analyse statique. 
L'étude de la liaison complète peut être effectuée à partir des formes temporelles des signaux. Il est également possible d'utiliser des outils propres à la caractérisation globale des liaisons numériques, tels que le diagramme de l'oeil ou le calcul du taux d'erreur par bit $[2,3,9]$.

Le diagramme de l'oeil (annexe H.7.) consiste à superposer sur une période temporelle donnée le signal obtenu en bout de liaison, afin d'observer toutes les transitions logiques possibles $(0 \rightarrow 0 \rightarrow 0 \rightarrow 0,0 \rightarrow 0 \rightarrow 0 \rightarrow 1$, $0 \rightarrow 0 \rightarrow 1 \rightarrow 1,0 \rightarrow 0 \rightarrow 1 \rightarrow 0$, etc., $1 \rightarrow 1 \rightarrow 1 \rightarrow 1$ ). Pour cela, on observe à l'oscilloscope le signal en bout de liaison en le synchronisant sur le signal d'horloge (ici la sortie du générateur de bits). L'ouverture de l'oeil renseigne alors sur la qualité de la liaison. L'outil de simulation permet ici de tracer directement l'oeil de la liaison. Il est ainsi demandé aux étudiants d'évaluer la qualité de la liaison globale en faisant varier plusieurs des paramètres pris en compte.

Le TEB (annexe H.6.) est quant à lui défini comme la probabilité d'erreur lors de la transmission d'un bit d'information. Seules de très faibles valeurs du TEB sont tolérées dans les systèmes de communications par fibre, typiquement TEB $<10^{-15}$. Le calcul du TEB s'effectue en général après évaluation du facteur Q, qui est lié au rapport signal à bruit électrique calculé en bout de liaison, c'est-à-dire au niveau du photodétecteur. TEB est alors donné par la relation suivante, où Erfc désigne la fonction d'erreur complémentaire :

$$
\mathrm{TEB}=\frac{1}{2} \operatorname{Erc}\left(\frac{Q}{\sqrt{2}}\right)
$$

À titre d'illustration, TEB $=10^{-9}$ requiert $Q=6$, tandis que TEB $=10^{-15}$ requiert $Q \approx 9$. Le logiciel COMSIS permet le calcul de $Q$ et donc de TEB, pour une liaison donnée. Cette information est précieuse car elle est plus quantitative que celle délivrée par le diagramme de l'oeil.

Ce type d'étude permet de répondre d'un point de vue global à des questions du type :

- $\quad$ pour une source laser donnée, modulée en courant à tel ou tel débit binaire (par exemple 1 Gbits. $\mathrm{s}^{-1}$ ), un amplificateur EDFA donné, et en considérant une fibre optique monomode standard et une photodiode idéale ayant une sensibilité S donnée, quelles sont les longueurs maximales des sections de fibre optique $L_{1}$ et $L_{2}$ tolérables pour un niveau donné de taux d'erreur par bit (TEB) ?

- $\quad$ que se passe-t-il si le débit binaire est multiplié par 10 ? (Fig. H8-1, annexe H.8.)

- $\quad$ si l'on souhaite effectuer sur le modèle décrit précédemment une liaison de $1000 \mathrm{~km}$ à un débit binaire de 10 Gbits.s $^{-1}$, quel type de diode laser faut-il adopter (ou à défaut faut-il avoir recours à un amplificateur externe, de type Mach-Zehnder par exemple), combien faut-il insérer d'amplificateurs EDFA de ligne et quelles sont les caractéristiques de chacun d'entre eux, quelles sont les longueurs des différentes sections de fibre, la dispersion chromatique est-elle rédhibitoire ou non, ... ?

\section{3. Étude d'une liaison WDM}

La figure 3 présente le schéma de la liaison étudiée par les étudiants. Celle-ci présente quatre diodes laser, un multiplexeur en longueur d'onde $4 \rightarrow 1$, une section de fibre optique monomode, un démultiplexeur en longueur d'onde $1 \rightarrow 4$ et un ensemble de quatre photodiodes idéales. 


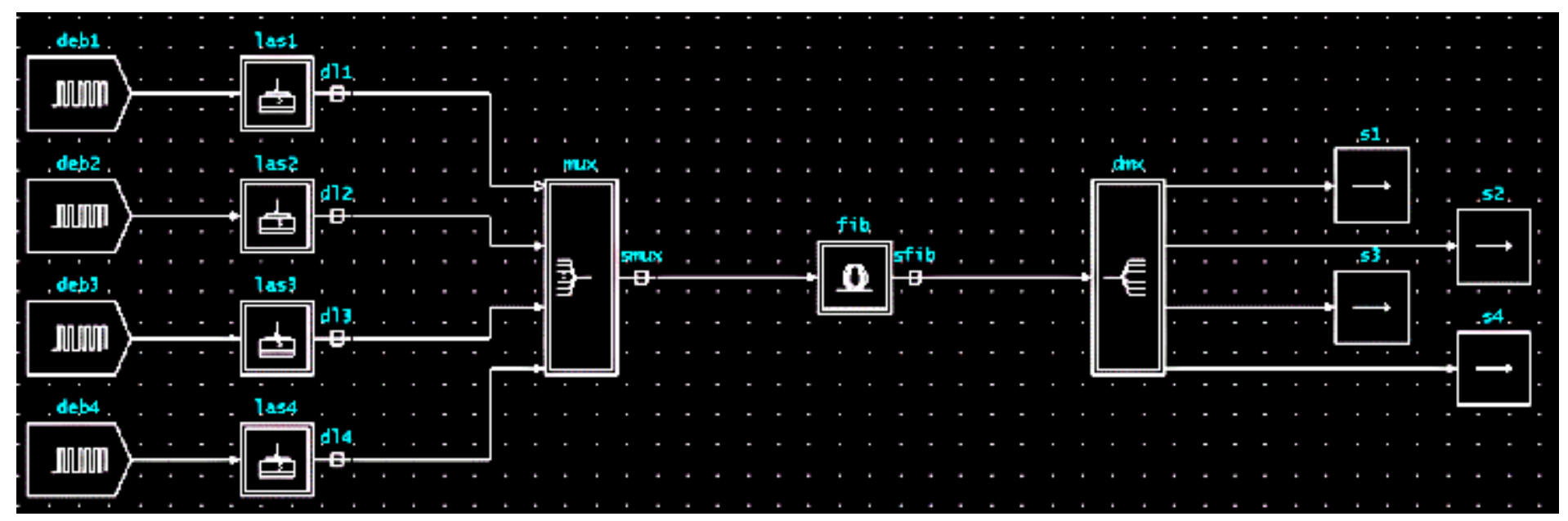

Fig. 3. Schéma de la liaison WDM.

Cette liaison très simple permet d'illustrer auprès des étudiants le principe des liaisons WDM. Du point de vue pratique, chacune des quatre longueurs d'ondes se voit affectée d'une couleur par le logiciel, ce qui facilite l'association à laquelle on a fréquemment recours entre couleur et longueur d'onde, et qui aide à l'analyse.

La description des multiplexeurs/démultiplexeurs en longueur d'onde est fournie aux étudiants (paramètres des MUX/DEMUX en longueurs d'onde, Fig. I-1, annexe I). Il leur est demandé d'analyser la signification des paramètres choisis et d'en prévoir l'impact sur le fonctionnement ultérieur de la liaison. Ces paramètres concernent essentiellement la longueur d'onde centrale, l'espacement en fréquence $\Delta v$, et l'atténuation entre canaux adjacents. Celle-ci s'exprime en $\mathrm{dB}$ et chiffre la diaphonie entre les canaux en longueurs d'onde. Il leur est alors demandé de choisir les longueurs d'onde des quatre diodes laser, leurs autres propriétés étant données. Aux étudiants donc, de déduire de l'espacement en fréquence $\Delta v=100 \mathrm{GHz}$, l'espacement en longueur d'onde entre canaux adjacents $\lambda=0,8 \mathrm{~nm}$. La longueur d'onde centrale du multiplexeur est de 1551,2 nm. L'espacement entre deux des quatre canaux étant de $0,8 \mathrm{~nm}$, la bande totale occupée s'étend sur $3 \times 0,8 \mathrm{~nm}=2,4 \mathrm{~nm}$, soit 1,2 nm autour de la longueur d'onde centrale. Celle-ci étant de $1551,2 \mathrm{~nm}$, les longueurs d'onde extrêmes sont $1550 \mathrm{~nm}$ et $1552,4 \mathrm{~nm}$. Les longueurs d'onde intermédiaires sont par ailleurs $1550,8 \mathrm{~nm}$ et $1551,6 \mathrm{~nm}$. La fibre optique est décrite quant à elle par un coefficient de pertes $\alpha=0,2 \mathrm{~dB} / \mathrm{km}$, un coefficient de dispersion chromatique $D_{\lambda}=17$ $\mathrm{ps} /(\mathrm{nm} . \mathrm{km})$, et une longueur $L=30 \mathrm{~km}$.

Lorsque tous les éléments de la liaison ont été décrits, on demande ensuite d'utiliser l'outil d'analyse statique afin de visualiser les propriétés des multiplexeur et démultiplexeur (caractéristique en longueur d'onde des MUX/DEMUX, Fig. I-2, annexe I). Cette étape permet de vérifier le choix qui a été fait des différentes longueurs d'onde des diodes laser. On demande en outre d'observer l'effet de la bande passante à $-3 \mathrm{~dB}$ des multiplexeur et démultiplexeur. La figure obtenue permet également de vérifier la réjection des canaux adjacents à $60 \mathrm{~dB}$ en-dessous du canal d'intérêt.

La liaison est ensuite analysée temporellement et spectralement en effectuant de proche en proche l'analyse des grandeurs le long de la liaison afin d'évaluer la qualité de la récupération des informations délivrées par les quatre générateurs pseudo-aléatoires (temporellement et spectralement) :

- On demande d'adopter des débits binaires différents sur les différentes voies de manière à illustrer la « transparence » de l'optique lors du multiplexage en longueur d'onde aux débits et aux formats des données : $1 \mathrm{Gbits.s}^{-1}$ sur la voie $1,2 \mathrm{Gbits.s^{-1 }}$ sur la voie 2, $3 \mathrm{Gbits.}^{-1}$ sur la voie 3 , et $4 \mathrm{Gbits.s^{-1 }}$ sur la voie 4 (forme temporelle des signaux optiques en sortie des généateurs pseudo-aléatoires, Fig. I-3, annexe I).

- Les signaux issus des diodes laser sont visualisés dans le temps (forme temporelle des signaux optiques en sortie des diodes laser, Fig. I-4a, annexe I) et analysés spectralement (densité spectrale de puissance des signaux optiques en sortie des diodes laser, Fig. I-4b, annexe I).

- Le spectre optique du signal issu du multiplexeur permet d'illustrer la superposition qui est opérée par multiplexage en longueur d'onde (densité spectrale de puissance du signal optique en sortie de multiplexeur, Fig. I-5a, annexe I). La largeur spectrale des signaux optiques est visible sur le spectre de ce signal multiplexé. Temporellement, ce signal ne contient aucune information d'interprétation aisée en raison des battements qui s'effectuent entre les différentes longueurs d'onde (forme temporelle du signal optique en sortie de multiplexeur, Fig. I-5b, annexe I). 
- En sortie de fibre optique, la forme du spectre est modifiée par les propriétés de dispersion chromatique de la fibre optique (densité spectrale de puissance du signal optique en sortie de la section de fibre, Fig. I-6a, annexe I). Pour s'en persuader, il suffit de reprendre la simulation de la liaison en annulant le coefficient de dispersion chromatique de la fibre (densité spectrale de puissance du signal optique en sortie d'une même section de fibre non-dispersive, Fig. I-6b, annexe I).

- Après démultiplexage, on observe le spectre de chacun des signaux de sortie $s_{1}, s_{2}, s_{3}$ et $s_{4}$. On observe sur ces courbes la réjection imparfaite des canaux WDM adjacents (densité spectrale de puissance des signaux optiques en sortie dedémultiplexeur, signaux séparés, Fig. I-7a, annexe I).

- Pour finir, on observe ces mêmes sorties dans le domaine temporel (forme temporelle des signaux optiques en sortie de démultiplexeur, signaux séparés, Fig. I-7b, annexe I). On observe en comparant les signaux de sortie et d'entrée que l'information est globalement conservée après multiplexage en longueur d'onde, propagation, démultiplexage, et détection.

Cette première série d'observations est complétée par d'autres simulations, où l'on fait varier la longueur de la liaison, les débits binaires des diodes laser ou leurs propriétés, etc. Dans chacun des cas, on vérifie par un diagramme de l'oeil sur chacune des quatre sorties que l'information est correctement transmise ou non.

\section{Conclusion}

Cet article présente une expérience menée à l'université Paris-Sud pour l'enseignement des télécommunications optiques au sein de la maîtrise EEA et du DESS Systèmes Électroniques d'Orsay. Un logiciel commercial (COMSIS), permettant une description fonctionnelle des chaînes de communication par fibre optique, est utilisé au cours de séances de TD/TP sur cette thématique. L'apport de ce type d'approche est de permettre une étude globale des liaisons simples ou mutiplexées en longueur d'onde (WDM), en mettant l'accent sur les principales limitations introduites par les différents composants optiques (fibre, source laser, amplificateur optique, etc.).

Dans un domaine pluridisciplinaire, faisant intervenir l'optique, les communications numériques, la physique des dispositifs à semiconducteurs, des lasers et des amplificateurs optiques, et où le matériel est extrêmement coûteux, ce type d'outil nous est apparu intéressant. L'expérience menée a été bien ressentie par les étudiants et leur a permis d'appréhender les systèmes de transmission optiques de manière assez souple et graduelle. Cette expérience a été complétée cette année par la mise en place de travaux pratiques mettant en jeu du matériel optique.

\section{Références bibliographiques}

[1] Logiciel COMSIS : http://www.ipsis.com (consulté le 27 mars 2003)

[2] Z. Toffano, Optoélectronique, Composants photoniques et fibres optiques (Ellipses, Technosup:Supelec, 2001)

[3] M. Joinot et I. Joinot, Télécommunications par fibres optiques (Dunod, 1996)

[4] P. Lecoy, Télécoms sur fibres optiques $2^{\mathrm{me}}$ ed. (Hermes, réseaux et télécommunications, 1997)

[5] E. Rosencher et B. Vinter, Optoélectronique (Masson, 1998)

[6] F. Cerf, Les composants optoélectroniques (Hermes Sciences, 2000)

[7] H.J.R. Dutton, Understanding Optical Communications $1^{\mathrm{re}}$ (presses IBM, 1998)

[8] N. Grote et H. Venghaus, Fiber-Optic Communication Devices (Springer, 2001)

[9] G. P. Agrawal, Fiber-Optic Communication Systems, $2^{\text {me }}$ ed. (Wiley, 1997)

[10] A.E. Willner, Mining the optical bandwidth for a terabit per second, IEEE Spectrum (apr. 1997)

[11] M.S. Borella, J.P. Jue, D. Banerjee, B. Ramamurthy et B. Mukherjee, Optical Components for WDM Lightwave Networks, Proceedings of the IEEE, 85 (8) (1997) 1274-1307. 


\section{Annexes}
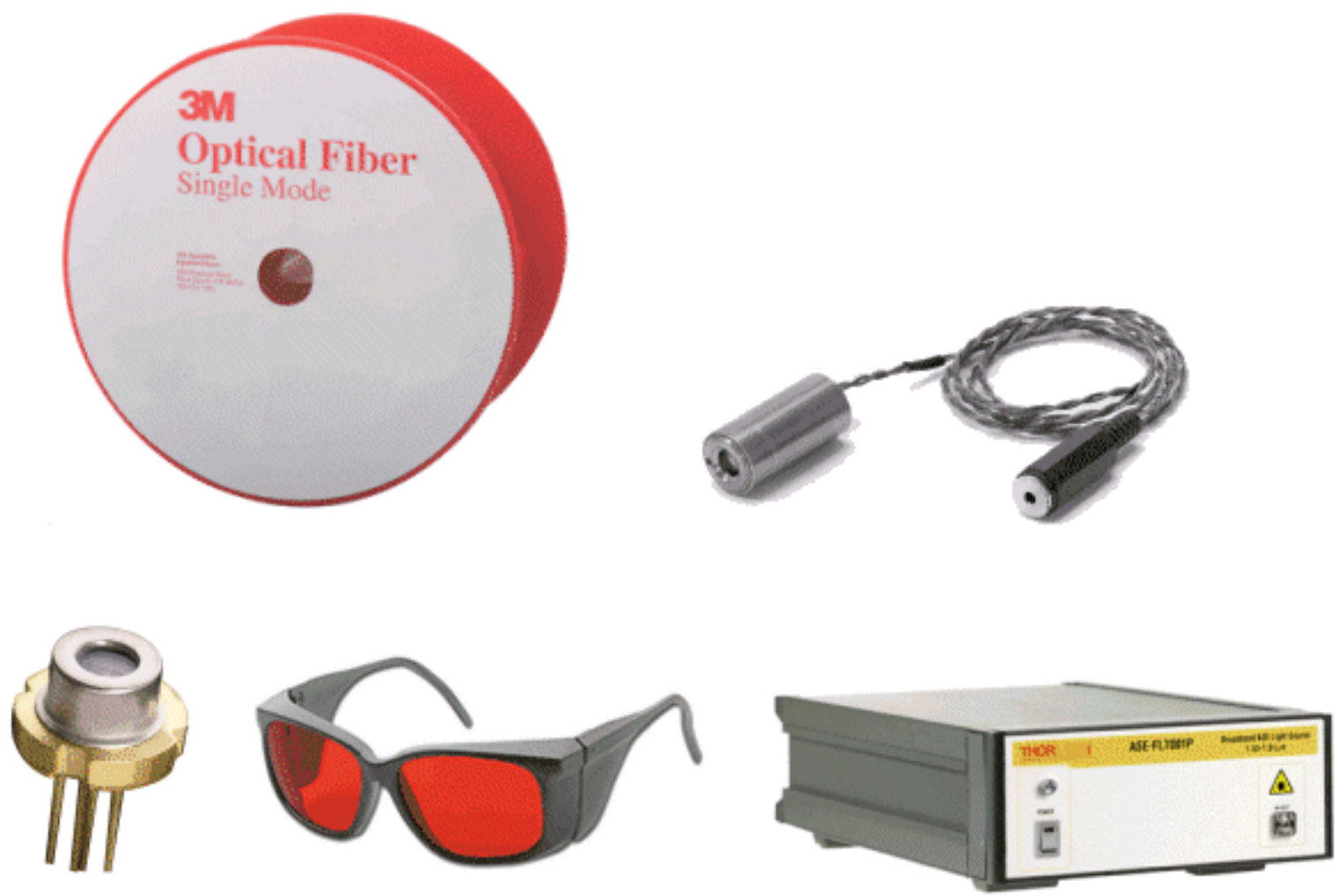

Source : images de matériel Optoprim-Thorlabs (http://www.optoprim.com/ et http://www.thorlabs.com/).

\section{Annexe A. Historique des communications par fibre optique}

Depuis l'apparition des transistors après la seconde guerre mondiale, les technologies associées aux communications ont progressé de manière spectaculaire. Depuis lors, la vitesse de fonctionnement des circuits électroniques double sensiblement tous les 18 mois, en raison de la miniaturisation toujours plus poussée des composants (loi de Moore). Les télécommunications ont profité très largement de cette évolution : elle a rendu possible la diffusion hertzienne d'ondes radio (porteuse de l'ordre de $100 \mathrm{MHz}$ en modulation de fréquence) et de télévision $(100 \mathrm{MHz}-1 \mathrm{GHz}$ ), le développement de la téléphonie mobile (porteuses de l'ordre du GHz), les communications par satellites (autour de $10 \mathrm{GHz}$ ), et les communications par fibre optique.

Parmi cet éventail, la technologie des transmissions par fibre optique est apparue très tardivement. Au début des années 1970, une première évolution favorable a résulté de la conjonction de deux facteurs : la fabrication de fibres optiques en silice à faibles pertes et la maîtrise des diodes laser à semiconducteurs. La commercialisation, au début des années 1990, des amplificateurs optiques à fibre dopée à l'Erbium (Erbium Doped Fiber Amplifier ou EDFA) a été ensuite fondamentale, car elle a permis la mise en place de liaisons « tout-optiques » de plusieurs milliers de kilomètres, sans régénération électronique des informations. Enfin, le développement, depuis la deuxième moitié des années 1990, de la technologie du multiplexage en longueur d'onde (Wavelength Division Multiplexing ou WDM) a conduit à un accroissement exponentiel de la capacité de transmission des fibres optiques, qui atteint d'ores et déjà $10 \mathrm{Tbits}^{-1} \mathrm{~s}^{-1}$, soit environ trois ordres de grandeur de plus que les systèmes électroniques les plus rapides. Les données, essentiellement traitées sous forme numérique, sont tout d'abord multiplexées temporellement (TDM : Time Division Multiplexing), à des débits normalisés par l'Union Internationale des Télécommunications (environ 2,5 Gbits. $\mathrm{s}^{-1}, 10 \mathrm{Gbits}^{-1} \mathrm{~s}^{-1}, 40 \mathrm{Gbits}^{-1} \mathrm{~s}^{-1}$ ), puis multiplexées en longueur d'onde (WDM). En 2000, environ 80 millions de kilomètres de fibre optique monomode ont été installés dans le monde. 

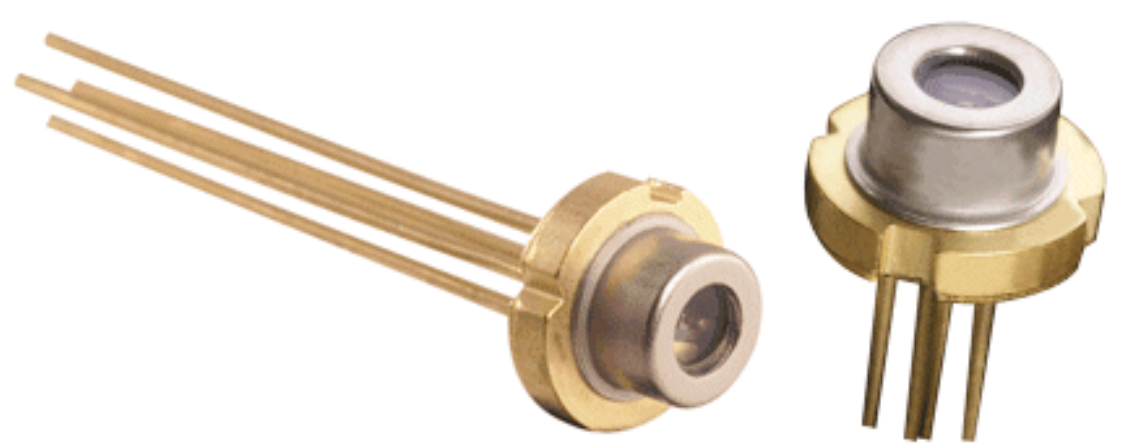

Fig. B-1. Exemples de diodes laser en boîtier.

Source : images de matériel Optoprim-Thorlabs (http://www.optoprim.com/ et http://www.thorlabs.com/).

Une diode laser (DL) est un oscillateur dans le domaine optique. L'obtention d'un effet laser est obtenue par l'action combinée d'un mécanisme degain optique et la sélection de certaines fréquences ou longueurs d'onde par le biais d'une cavité optique (par exemple de type Fabry-Perot) [2-4].

Le gain optique est obtenu, au sein de matériaux semiconducteurs à bande interdite directe (alliages III/V), par la possibilité de favoriser l'émission stimulée de photons par rapport au mécanisme d'absorption dès lors que la population en électrons de la bande de conduction est plus importante que celle de la bande de valence. On parle alors d'inversion des populations électroniques. Cette situation, au demeurant fort inhabituelle (!), est rendue possible par l'injection d'un niveau de courant de dépassant un certain seuil, dont la valeur dépend de la structure de la DL : c'est le courant de seuil $I_{S}$ de la DL. En-dessous de ce seuil, l'émission de lumière est obtenue par émission spontanée de photons, comme dans les diodes électroluminescentes (DEL). Au-delà, l'émission stimulée domine et la puissance optique délivrée par la DL augmentent à peu près linéairement avec l'écart de courant injecté par rapport au seuil, avec une pente de conversion de quelques $0,1 \mathrm{~W} / \mathrm{A}$.

La valeur du courant de seuil de la DL dépend beaucoup de sa structure (typiquement de quelques mA à quelques dizaines de $\mathrm{mA}$ ), mais il varie exponentiellement avec la température selon la loi :

$$
I_{z}\left(T_{2}\right)=I_{z}\left(T_{1}\right) \times \exp \left(\frac{T_{2}-T_{1}}{T_{0}}\right)
$$

où $T_{0}$ dépend du matériau utilisé pour l'émission.

Ces résultats sont illustrés par la figure suivante, qui présente un réseau de caractéristiques $P(I)$ obtenues pour différentes températures de fonctionnement. On y note que l'ordre de grandeur de la puissance optique délivrée par les DL (télécoms) est le mW.

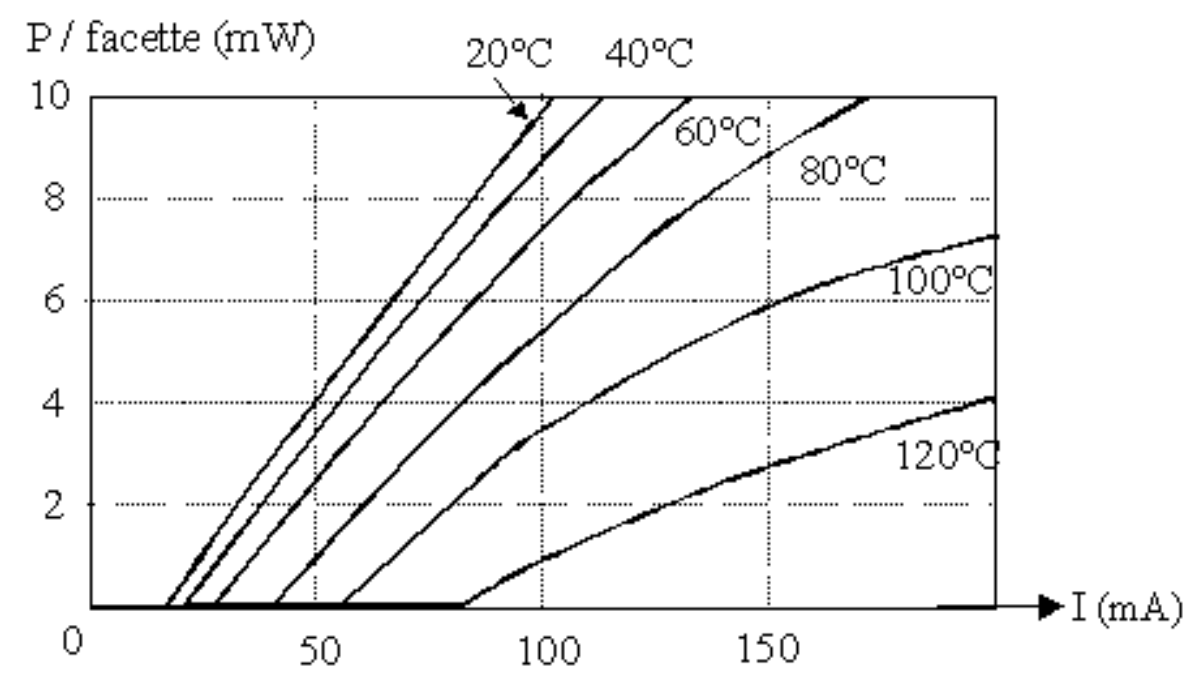


Fig. B-2. Exemple typique de caractéristique statique : puissance optique en fonction du courant injecté.

\section{Annexe C. Caractéristiques dynamiques des diodes laser}

\section{Utilisation des diodes laser pour les communications numériques}

Les diodes laser (DL) sont utilisées pour coder de l'information sous forme optique que l'on injecte ensuite dans la fibre optique.

Dans le cadre des communications numériques par fibre, on module courant de la diode laser entre deux niveaux $I_{P}$ et $I_{M}$, dont le plus faible est choisi au dessus du courant de seuil $I_{S}$ de la DL.

\section{Temps de retard et oscillations de relaxation}

Idéalement, la forme temporelle des impulsions optiques délivrées devraient être parfaitement fidèle à la succession des bits d'information pilotant le circuit de commande en courant. Cela n'est bien sûr pas le cas, et l'est d'autant moins que le débit binaire est élevé (et donc que la durée des impulsions successives est courte) (Fig. C-1).

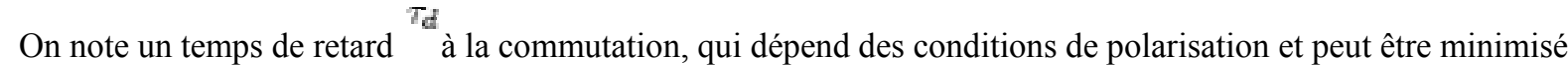
par une polarisation en continu $\left(I_{P}>I_{S}\right)$. On note également la présence d'oscillations de « relaxation », dont l'origine physique tient à la mise à l'équilibre des populations d'électrons et de photons (populations couplées) sous l'effet d'un changement du niveau de courant.

Ces oscillations sont amorties pour des courants de polarisation plus élevés. L'ordre de grandeur de ${ }^{T_{d}}$ est inférieur à la nanoseconde et la fréquence des oscillations de relaxation est de quelques GHz. Les DL peuvent ainsi être modulées en courant jusqu'à quelques Gbits. $\mathrm{s}^{-1}$ (cela dépend beaucoup de la structure de la DL).

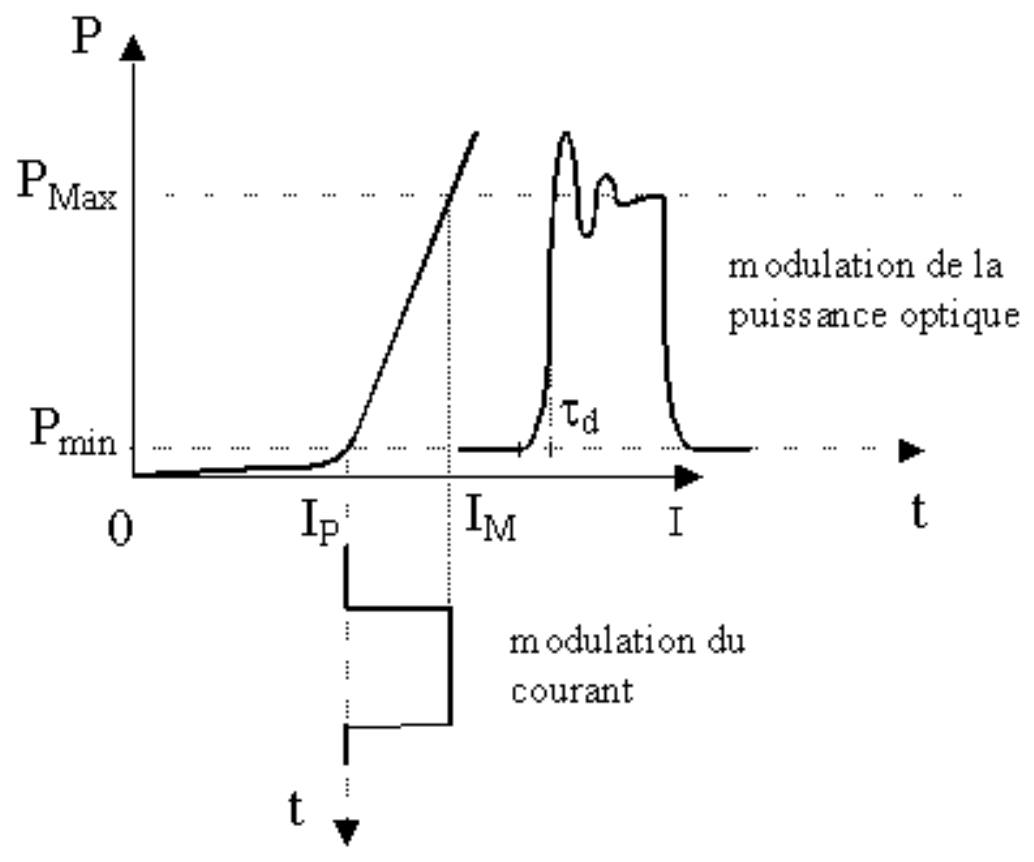

Fig. C-1. Oscillations de relaxation de la puissance optique délivrée sous l'effet d'une modulation d'amplitude du courant de la diode laser entre deux valeurs $I_{P}$ et $I_{M}$. 


\section{Largeur spectrale}

En plus de l'altération de la forme temporelle des impulsions, la lumière émise par les diodes laser (DL) possède un spectre non-monochromatique. La conséquence en est l'étalement des impulsions optiques au cours de la propagation en raison de la dispersion chromatique de la fibre optique.

Plusieurs raisons expliquent ce résultat. En premier lieu, le codage sous forme numérique d'informations par le biais d'une modulation d'amplitude de la puissance optique (modulation de type ASK appliquée à la porteuse optique), conduit nécessairement à un élargissement spectral autour de la longueur d'onde de la porteuse. Par ailleurs, les DL possèdent une largeur spectrale statique, c'est-à-dire en l'absence de toute modulation, qui est liée à la structure de la DL (cavité optique de type Fabry-Perot, DFB, etc.).

Enfin, lors de la modulation du courant des DL, la fréquence d'émission varie légèrement. On parle de « chirp » (dynamique). Ce phénomène est lié à la variation de la fréquence du mode, qui est une fonction du courant injecté (modulation FM non souhaitée). On définit l'efficacité de modulation FM en MHz/mA (habituellement 50 à $500 \mathrm{MHz}$ par mA). La source laser est d'autant moins monochromatique que le « chirp » est élevé.

\section{Bruit d'intensité optique (Relative Intensity Noise ou RIN)}

Le bruit d'intensité optique est provoqué par l'émission spontanée de photons qui a lieu au sein de la cavité laser. Le spectre de ce bruit n'est pas «plat » et sa forme dépend du courant de polarisation. Des valeurs très basses de RIN sont nécessaires dans les liaisons optiques, typiquement $<-100 \mathrm{~dB} / \mathrm{Hz}$. 

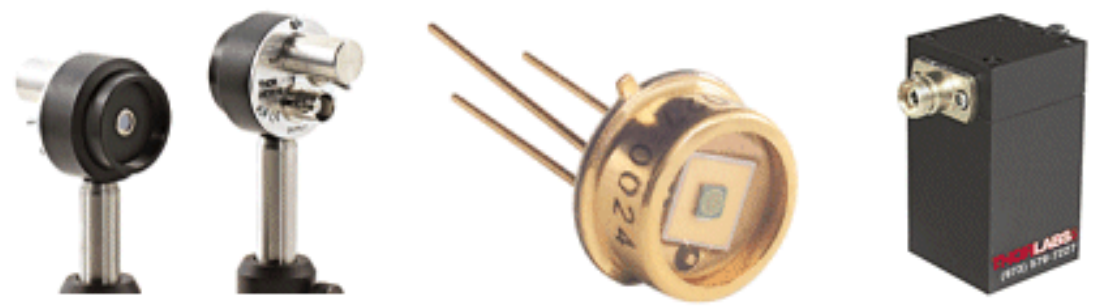

Fig.D-1. Exemples de photodétecteurs fibrés et non fibrés.

Source : images de matériel Optoprim-Thorlabs (http://www.optoprim.com/ et http://www.thorlabs.com/)

Le facteur essentiel qui détermine le rendement d'un photodétecteur est le coefficient d'absorption $\alpha$ qui est caractéristique du matériau utilisé (Fig. D-2). Ce coefficient d'absorption dépend bien sûr de la longueur d'onde de la lumière. Les autres caractéristiques sont les suivantes :

- la longueur d'onde de coupure $\lambda_{c}$ au-delà de laquelle le matériau devient transparent au rayonnement est déterminée par l'énergie de bande interdite $E_{G}$ du semiconducteur :

$A_{c}(\mathrm{~mm})=\frac{1,24}{\mathrm{E}_{\mathrm{G}}(\mathrm{eV})}(\Rightarrow 1,1$ m pou Si $)$

- le rendement quantique $\left({ }^{\eta_{Q}}\right.$ est le rapport du nombre de paires de porteurs « photocréées et collectées » au nombre de photons incidents.

- la sensibilité $S(\lambda)$ de la photodiode est le rapport du photocourant $I_{p h}$ au flux énergétique (ou puissance optique) reçu.

Ces grandeurs sont reliées par les relations suivantes, où $e$ représente la charge élémentaire de l'électron :

$$
n_{Q}=\frac{(I / e)}{\left(P_{\mathrm{s} p t} / h \nu\right)} \quad S(\lambda)=\frac{I_{p h}}{P_{\mathrm{opt}}}=\eta Q \frac{e \cdot \lambda}{h \cdot c}
$$




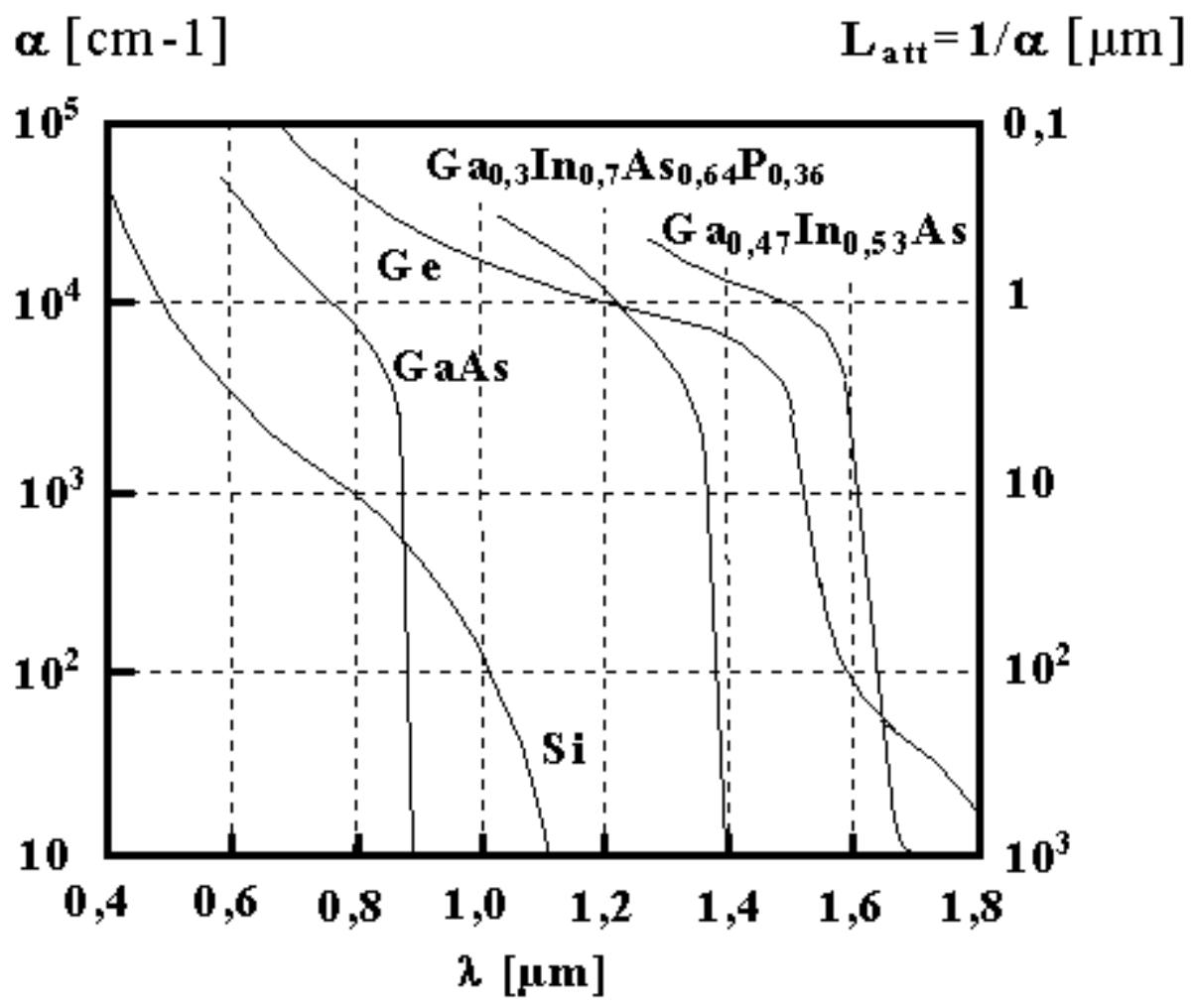

Fig.D-2. Absorption des matériaux semi-conducteurs.

La puissance optique $P_{o p t}$ décroît selon une loi exponentielle dans laquelle intervient le coefficient $\alpha$ et où $x$ désigne la profondeur de pénétration de la lumière dans le semiconducteur : $P_{\mathrm{opt}}(x)=P_{\mathrm{opt}}(0) \times \exp (-\alpha I)$ 


\section{Annexe E. Fibre optique}
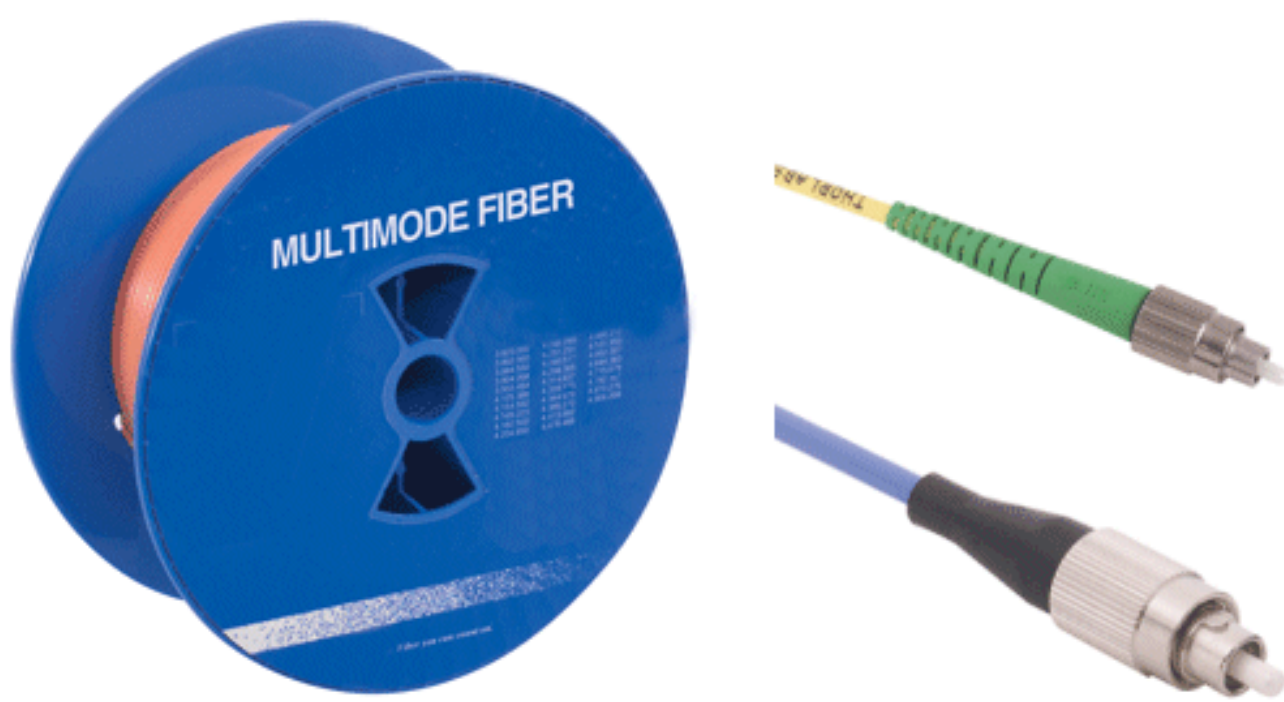

Fig.E-1. Rouleau de fibre optique multimode (à gauche); exemples de connecteurs (à droite).

Source : images de matériel Optoprim-Thorlabs (http://www.optoprim.com/ et http://www.thorlabs.com/)

\section{Différents types de fibres optiques}

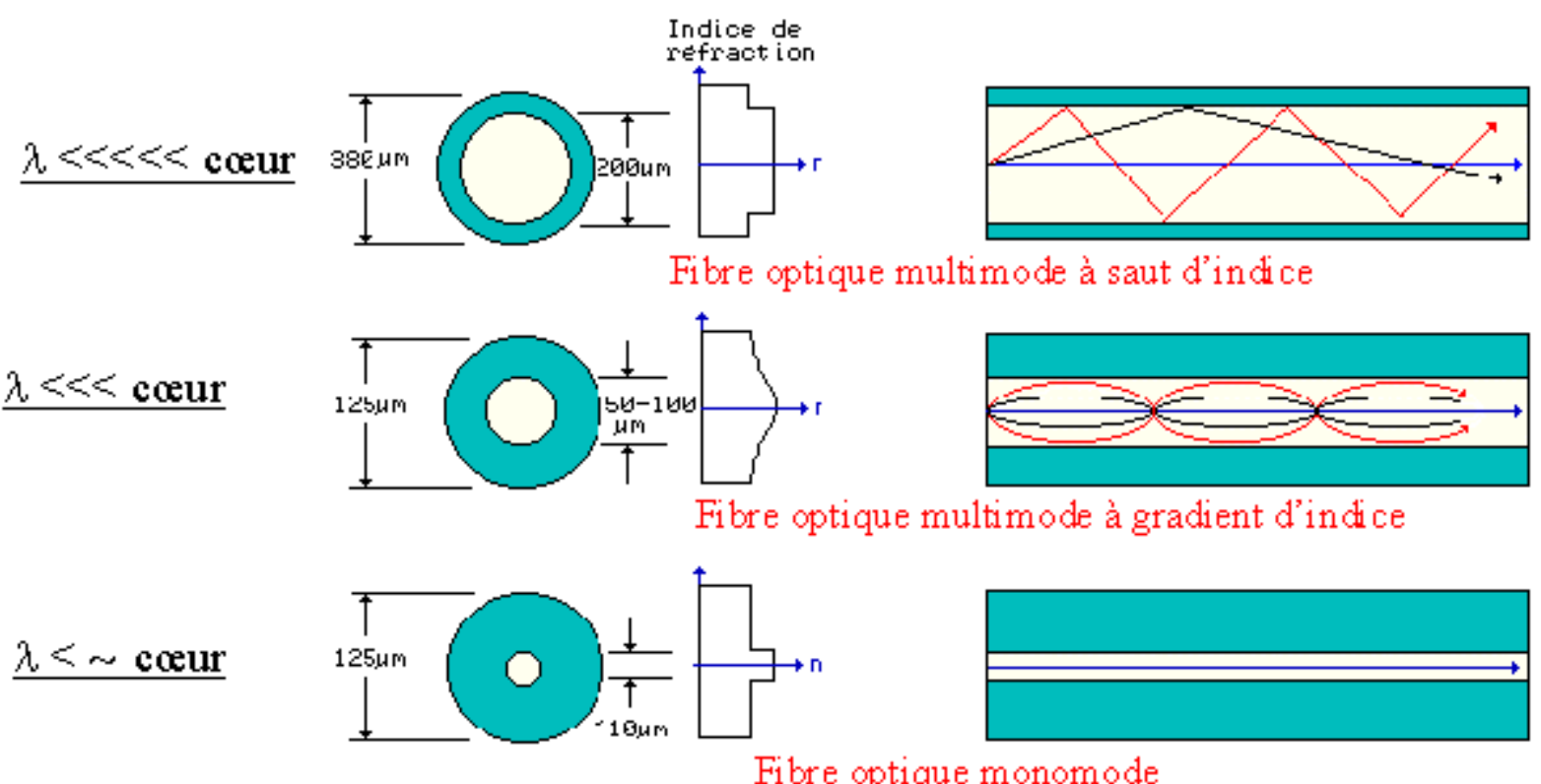

Fig.E-2. Classsification des fibres optiques.

Source : http://www.unige.ch/dinf/jfl/fibre/

L'étude rigoureuse des phénomènes de propagation dans les fibres optiques repose sur la résolution des équations de Maxwell. L'inconvénient de cette approche est sa lourdeur mathématique (guides à section cylindrique, dans lesquels les solutions exactes se développent à partir de fonctions de Bessel $[2,3,7,9]$ ). On montre ainsi qu'à une longueur d'onde donnée, il existe en général plusieurs modes de propagation de la lumière au sein de la fibre.

Pour un profil d'indice de réfraction donnée entre le coeur et la gaine, le nombre de modes dépend essentiellement du rapport entre le diamètre $d$ du coeur et la longueur d'onde $\lambda$ de la lumière. Dans la limite où $d / \lambda$ est suffisamment faible, le nombre de modes $\mathrm{Nb}$ est restreint à 1 . On distingue ainsi classiquement les fibres 
multimodes $(\mathrm{Nb}>1)$ des fibres monomodes $(\mathrm{Nb}=1)$. Il est à noter que le profil d'indice de réfraction n'est pas nécessairement abrupt. Parmi les fibres multimodes, on distingue ainsi les fibres à saut d'indice des fibres à gradient d'indice, comme le montre la figure E-2.

\section{Pertes de propagation de fibres optiques}

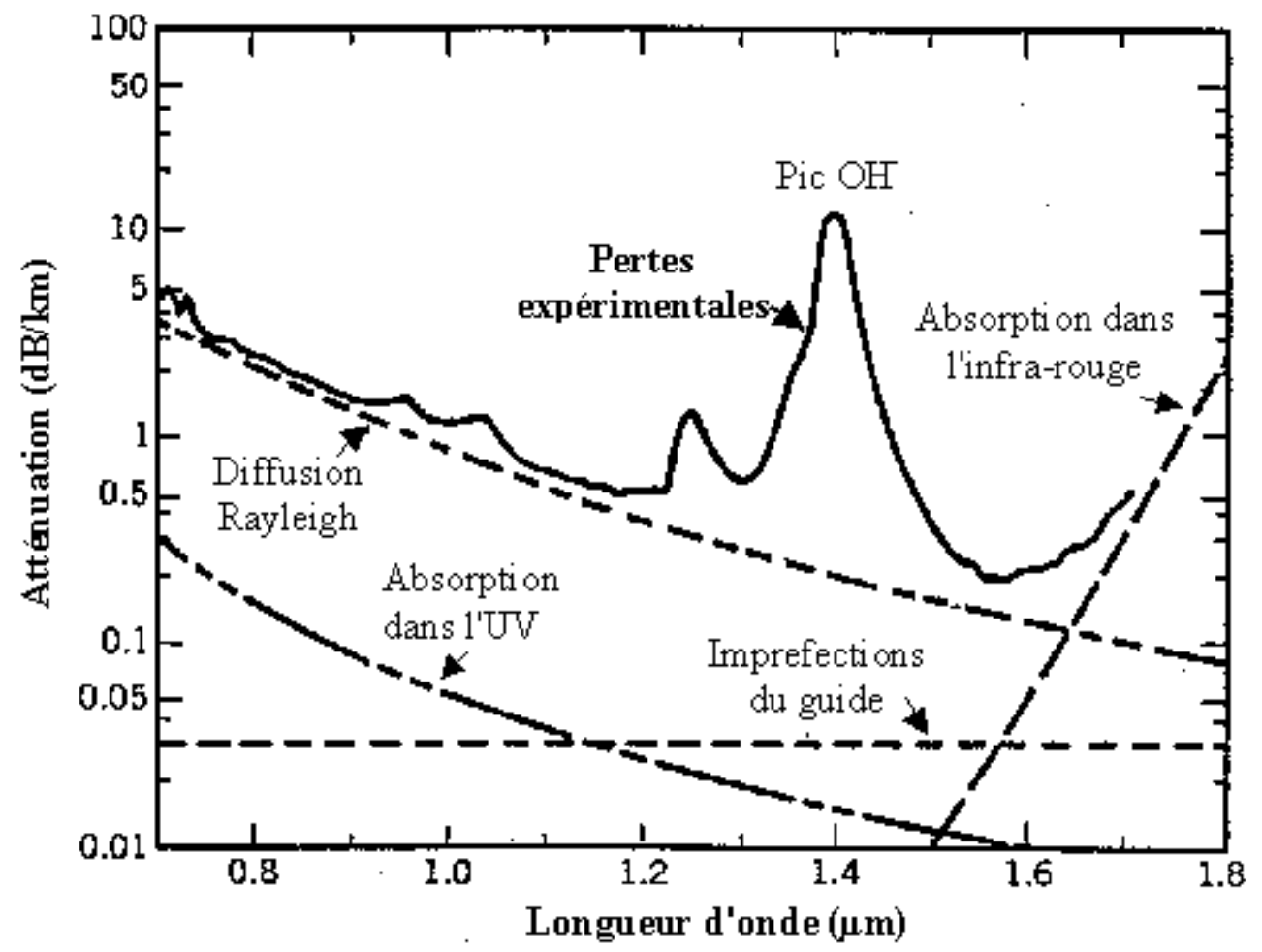

Fig.E-3. Pertes de propagation des fibres optiques standard.

L'atténuation de la fibre optique (en silice) est liées à plusieurs causes. D'une part, la lumière est partiellement absorbée par la matière dans l'infra-rouge et dans l'ultra-violet. Par ailleurs, une quantité résiduelle d'eau, incorporée sous forme d'ions $\mathrm{OH}^{-}$au cours de la fabrication, peut être responsable d'un pic d'atténuation, bien visible sur la courbe ci-contre au voisinage de 1,4 $\mu \mathrm{m}$. Par ailleurs, les inhomogénéités de matière (matrice de silice), sont responsables d'une diffusion partielle de la lumière, appelée diffusion Rayleigh. La courbe de la figure E-3 fait apparaître des longueurs d'onde privilégiées. Au voisinage de $\lambda=1,31 \mu \mathrm{m}$, on note la présence d'un minimum relatif des pertes de propagation, tandis qu'au voisinage de $\lambda=1,55 \mu \mathrm{m}$, les pertes sont les plus faibles. C'est la raison pour laquelle les télécommunications par fibre optique s'effectuent principalement autour de cette dernière longueur d'onde. L'atténuation est alors de l'ordre de $0,2 \mathrm{~dB} / \mathrm{km}$, ce qui signifie que la lumière peut parcourir $100 \mathrm{~km}$ avant que son niveau de puissance ne soit divisé par 100. 


\section{Dispersion intermodale}

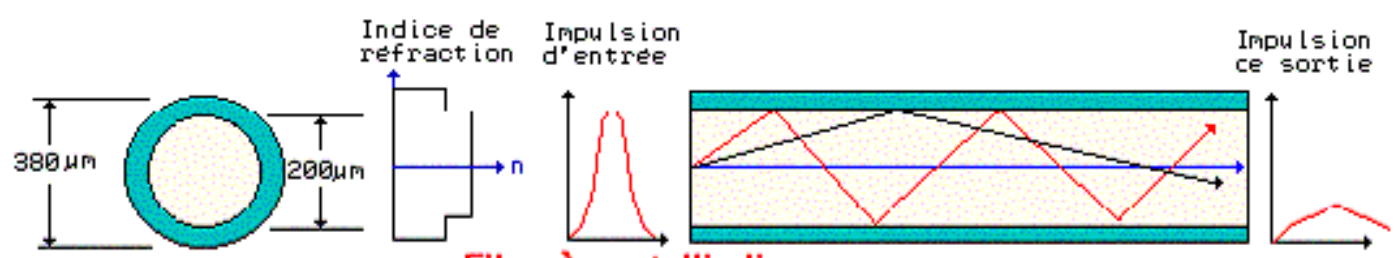

Fibre à saut d'indice
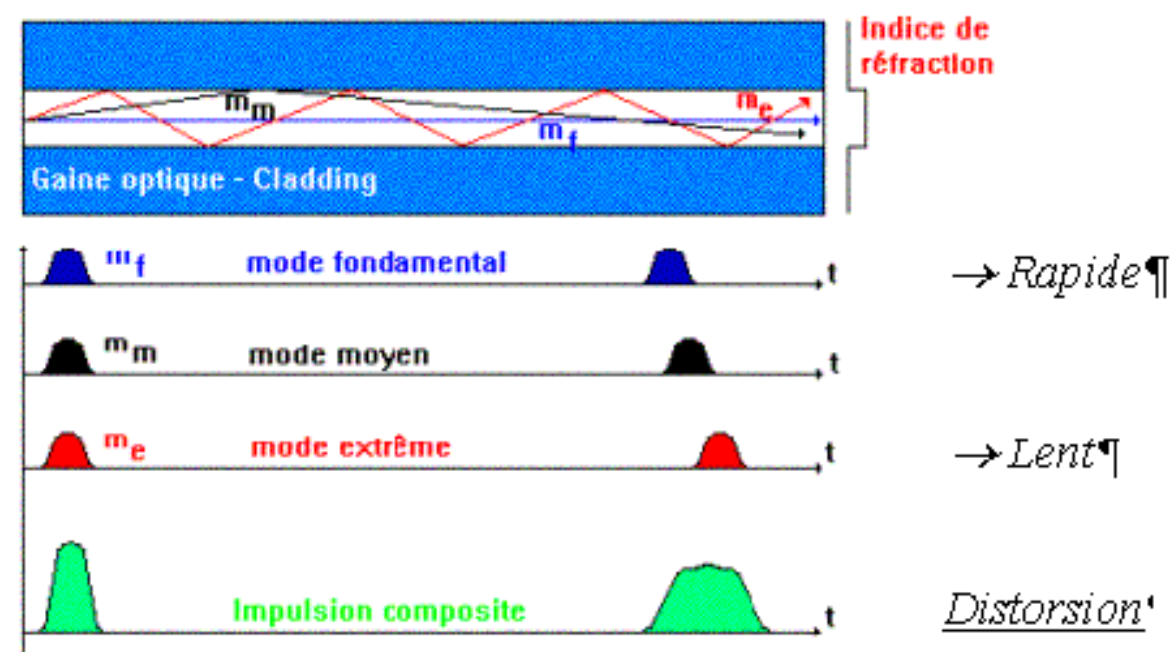

Fig.E-4. Déformation des impulsions de lumière dans les fibres optiques multimodes.

Source : http://www.unige.ch/dinl/jfl/fibre/

Dans le cas des fibres multimodes, la dispersion intermodale est la source principale de déformation temporelle des impulsions optiques codant l'information numérique. Comme ce type de fibre possède plusieurs modes de propagation, certains d'entre eux se propagent plus rapidement que d'autres. Si l'on adopte l'approche de l'optique géométrique (Fig. E-4), valable dans ce type de fibres où $\lambda<<$ diamètre de coeur, on peut dire que certains modes ou « rayons » se propagent davantage dans l'axe du guide que d'autres, et sont donc plus rapides que ces derniers. Une impulsion optique « composite », c'est-à-dire qui excite en entrée de fibre un grand nombre de modes, voit donc son énergie véhiculée par des « supports » de vitesses différentes. Il en résulte qu'après propagation selon une distance L donnée, la forme temporelle de l'impulsion est altérée. Dans le contexte des communications numériques par fibre, le phénomène de dispersion intermodale se traduit par un étalement des impulsions optiques, et limite le débit binaire D qu'il est possible de transmettre selon une loi du type :

Dmax $\times \mathrm{L}=$ Constante (de l'ordre de quelques centaines de MHz.km). Ce type de fibre optique est donc réservé à des applications courte distance. 


\section{Dispersion chromatique des fibres optiques monomodes}

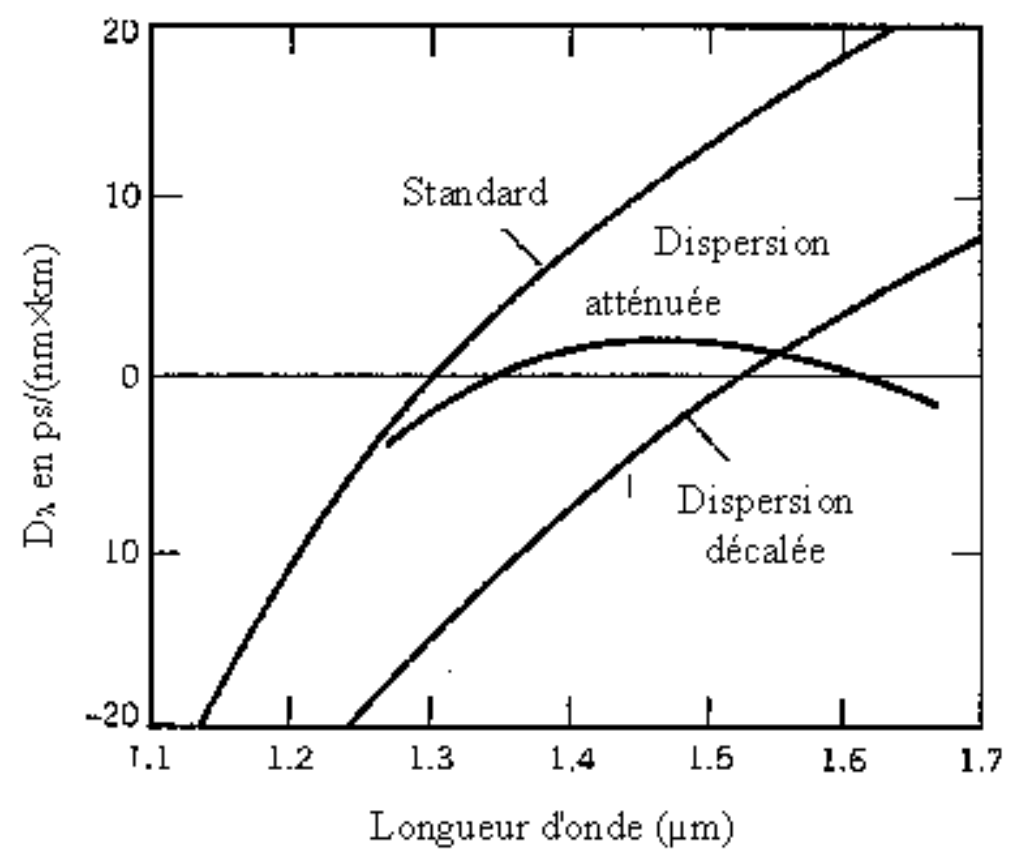

Fig.E-5. Coefficient de dispersion chromatique de plusieurs types de fibres optiques monomodes.

Contrairement au cas des fibres multimodes, les fibres optiques monomodes ne possèdent qu'un seul mode de propagation, pour une longueur d'onde donnée. Le problème est que la lumière injectée dans la fibre n'est pas monochromatique (!) en raison de la modulation de la porteuse optique nécessaire au codage de toute information, et aux propriétés physiques des émetteurs utilisés (diodes laser). Le spectre occupé est certes très étroit mais présente une certaine extension en longueur d'onde (par exemple 0,2 nm autour de $1550 \mathrm{~nm}$ ). Les différentes longueurs d'onde étant proches les unes des autres, la fibre reste monomode pour chacune de ces longueurs d'onde. Néanmoins, les propriétés de guidage font que certaines longueurs d'onde se propagent plus rapidement que d'autres. Il en résulte qu'une impulsion optique émise à l'entrée d'une section de fibre, qui est en fait un paquet d'ondes riche de nombreuses longueurs d'onde très proches, se déforme au cours de la propagation.

Ce type de dispersion, qui est appelée dispersion chromatique, est également présent dans le cas des fibres multimodes, mais elle y est négligeable devant la dispersion intermodale.

Dans une large mesure, il est possible de rendre compte de la dispersion chromatique en faisant intervenir le coefficient $D_{\lambda}$, dit de dispersion chromatique. Ce coefficient intervient dans le calcul de l'étalement des impulsions temporelles se propageant dans la fibre, comme indiqué précédemment.

La figure E-5 donne $D_{\lambda}$ pour plusieurs types de fibres optiques : fibre optique standard, pour laquelle $D_{\lambda}$ s'annule au voisinage de $\lambda=1,31 \mu \mathrm{m}$, fibre optique à dispersion atténuée, pour laquelle le profil d'indice de réfraction choisi permet d'obtenir de faibles valeurs de $D_{\lambda}$ dans une large plage de longueurs d'onde, et fibre à dispersion chromatique décalée, pour laquelle on annule $D_{\lambda}$ à la longueur de 1,55 $\mu \mathrm{m}$ (l'atténuation est alors minimale : $\mathrm{A}=0,2 \mathrm{~dB} / \mathrm{km})$. 


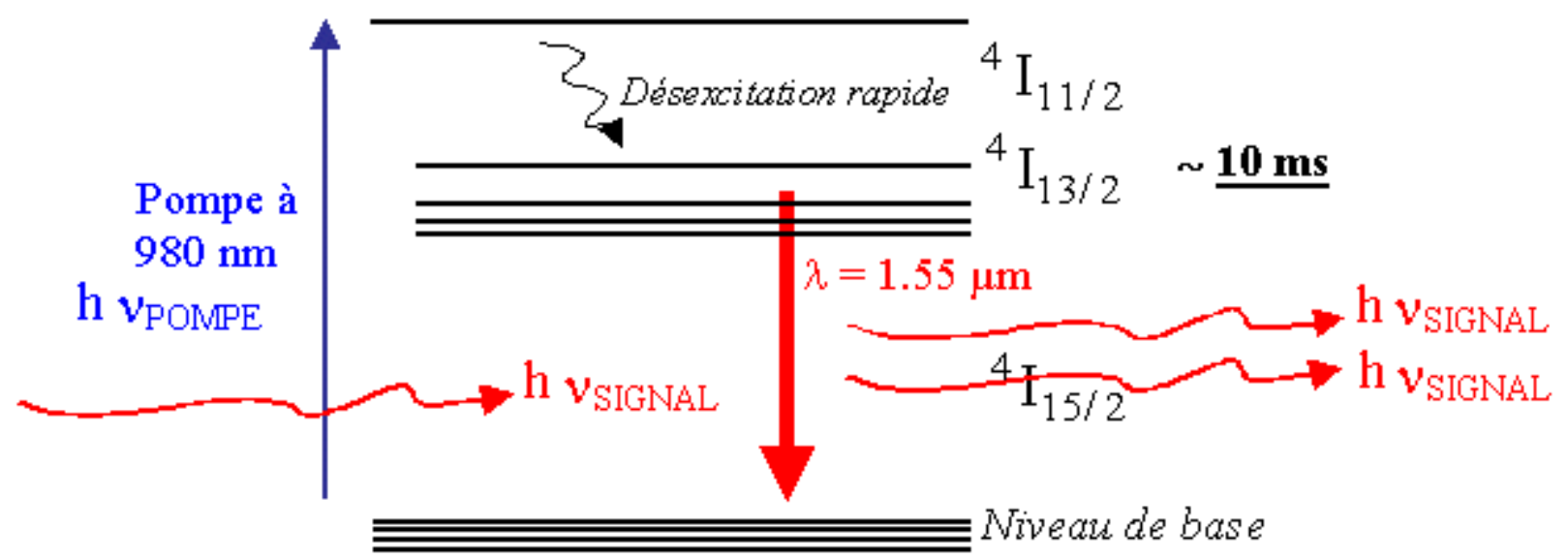

Fig.F-1. Schéma simplifié des niveaux en énergie intervenant dans le processus d'émission stimulée.

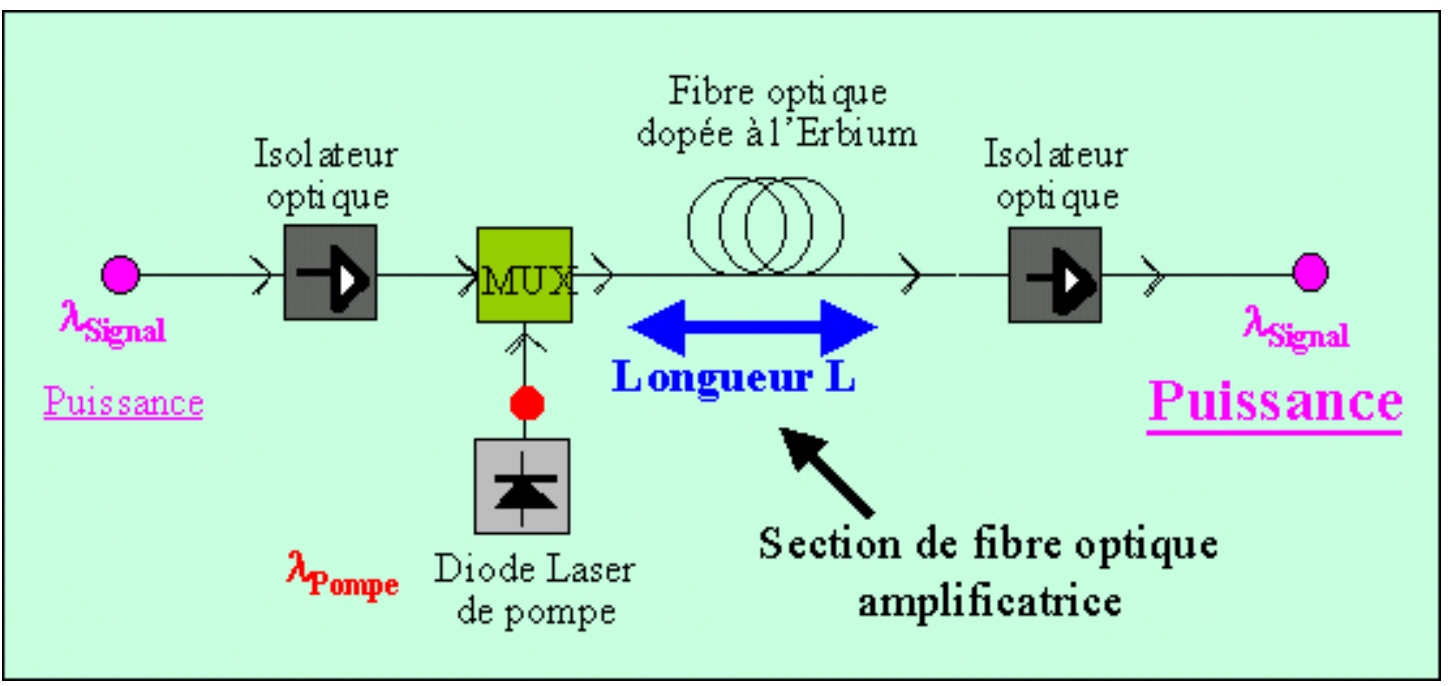

Fig.F-2. Schéma de principe de l'amplificateur à fibre dopée Erbium (EDFA).

\section{Annexe G. Multiplexage des signaux optiques}

Les signaux véhiculés par les fibres optiques sont très souvent multiplexés temporellement (TDM) et en longueur d'onde (WDM). Néanmoins, ceci n'a rien d'obligatoire et ces deux formes de multiplexage peuvent exister à l'exclusion l'une de l'autre.

Pour comprendre la nature de ces formes de multiplexage et leurs différences, il est utile d'adopter une double représentation temporelle et fréquentielle (fréquence ou longueur d'onde) des signaux. Les deux figures ci-contre illustrent le principe du TDM et celui du WDM.

\section{Multiplexage TDM}

Dans le cas du multiplexage temporel, l'information est constituée de différents signaux électroniques codés sous forme numérique qui sont combinés dans le domaine temporel par un multiplexeur électronique (circuit rapide dédié) pour obtenir un signal électronique de plus haut débit binaire. Par exemple, 16 signaux de débits binaires 155,52 $\mathrm{Mbits}^{-1} \mathrm{~s}^{-1}$ (débit normalisé STM-1) peuvent être multiplexés dans le temps pour obtenir un signal numérique de débit binaire 2,48832 Gbits.s ${ }^{-1}$ (débit normalisé STM-16). Sur la figure G-1(TDM), cette étape est schématisée par la présence de trois circuits électroniques et d'un multiplexeur électronique. 
Ce signal « haut débit » est utilisé pour la modulation du courant d'une diode laser. Le signal optique injecté dans la fibre ne présente donc qu'une unique fréquence (ou longueur d'onde), comme le montre la figure G-1 (et donc une unique couleur : le noir ici). Après propagation de ce signal dans une section de fibre de fibre, l'information « haut débit » est récupérée au moyen d'un photodétecteur. Un circuit électronique de démultiplexage temporel permet finalement la récupération des différents signaux « bas débit ».

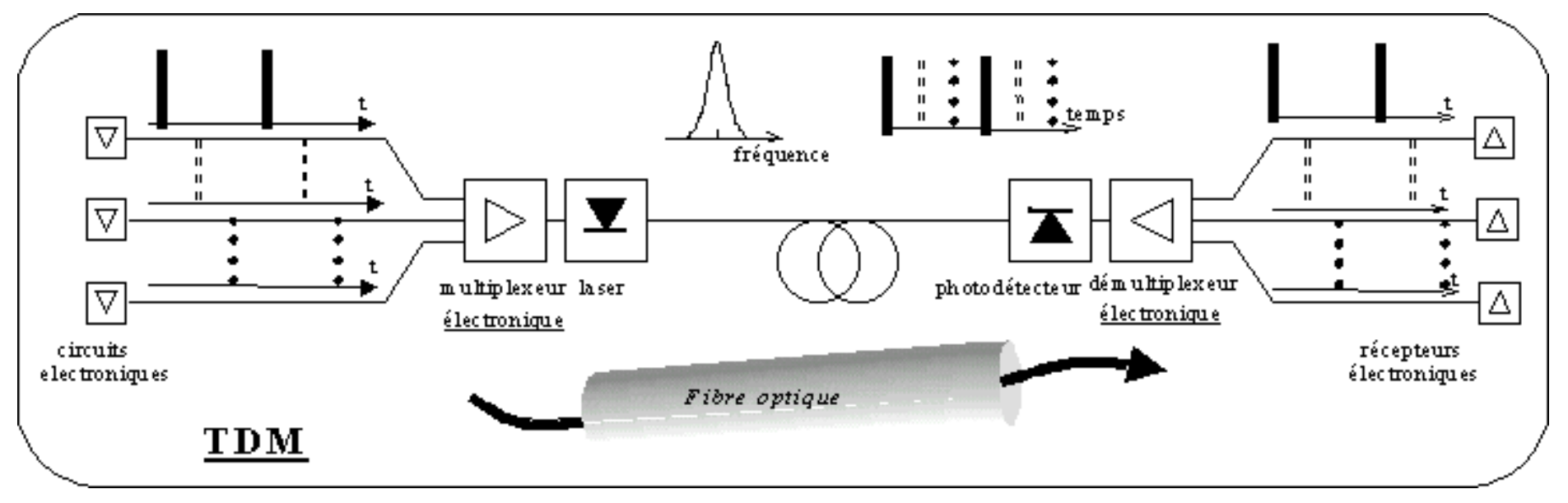

Fig.G-1. Schéma de principe de l'utilisation du démultiplexage temporel (TDM) dans les communications par fibre optique.

\section{Multiplexage WDM}

Dans le cas du multiplexage en longueur d'onde, on utilise un ensemble de diodes laser émettant à des fréquences (ou longueurs d'onde) très proches les unes des autres (quelques $0,1 \mathrm{~nm}$ au voisinage de $1550 \mathrm{~nm}$ ). Sur la figure G-2, ce point est illustré par l'utilisation de trois couleurs distinctes. Chaque diode laser est modulée en courant par un signal temporel de débit binaire et de format donné.

Un multiplexeur optique est utilisé pour combiner ces signaux dans le domaine optique, et ce indépendamment de la forme temporelle des signaux de modulation des diodes laser. Il s'agit d'un composant passif purement optique (par exemple un AWG [6-8]). La fibre, placée en aval comme support de propagation, véhicule un signal dont le spectre est riche de plusieurs canaux en longueurs d'onde (canaux WDM), comme illustré sur la figure G2. Dans la limite où les effets non-linéaires de la fibre peuvent être négligés, les différentes « couleurs » n'interagissent pas du tout entre elles, ce qui permet le transport de signaux de modulation aux formats et aux débits binaires quelconques les uns par rapport aux autres.

En sortie de fibre, un démultiplexeur optique permet de séparer les différentes « couleurs ». Un ensemble de photodétecteurs, en même nombre que les diodes laser, permet de restituer au niveau de chaque canal l'information sous forme électrique.

Il est à noter que rien n'empêche que chacun des signaux de modulation des diodes laser soit lui-même issu d'une étape de multiplexage temporel dans le domaine électronique. C'est même la situation la plus fréquente. Dans ce cas, après démultiplexage optique et photodétection, une étape de démultiplexage temporel permet la restitution des signaux temporels « bas débit ». 


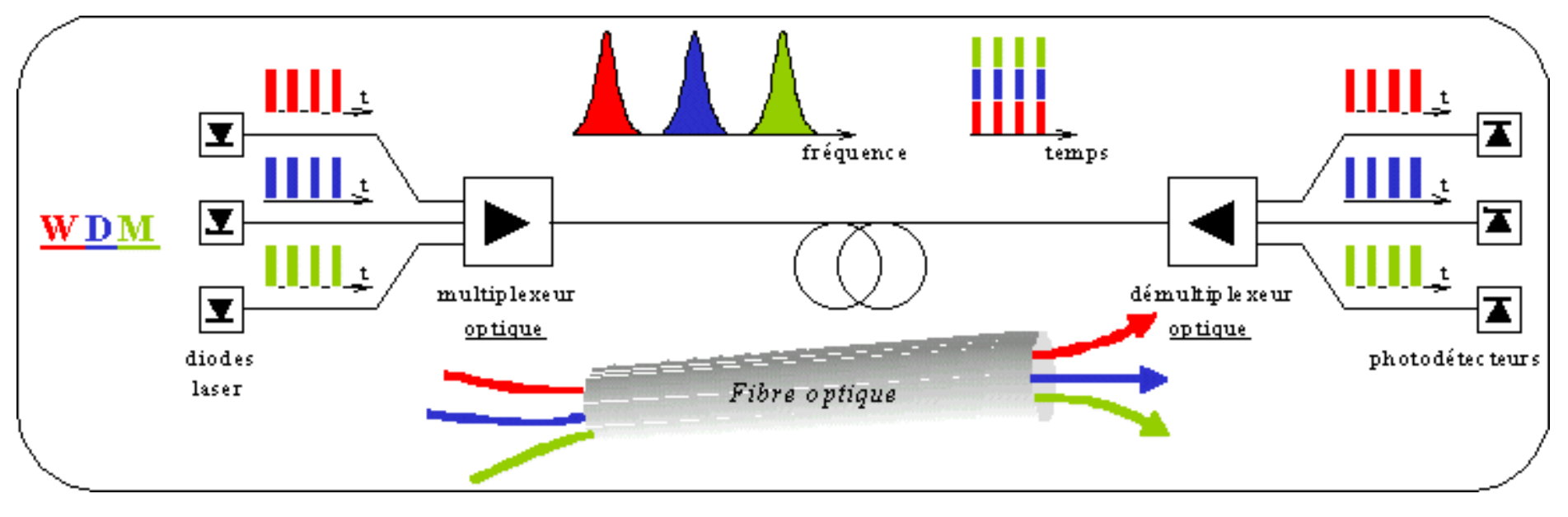

Fig.G-2. Schéma de principe de l'utilisation du démultiplexage en longueur d'onde (WDM) dans les communications par fibre optique.

Annexe H. Étude d'une liaison point à point mono-longueur d'onde

H.1. Caractéristiques des éléments de la liaison point à point
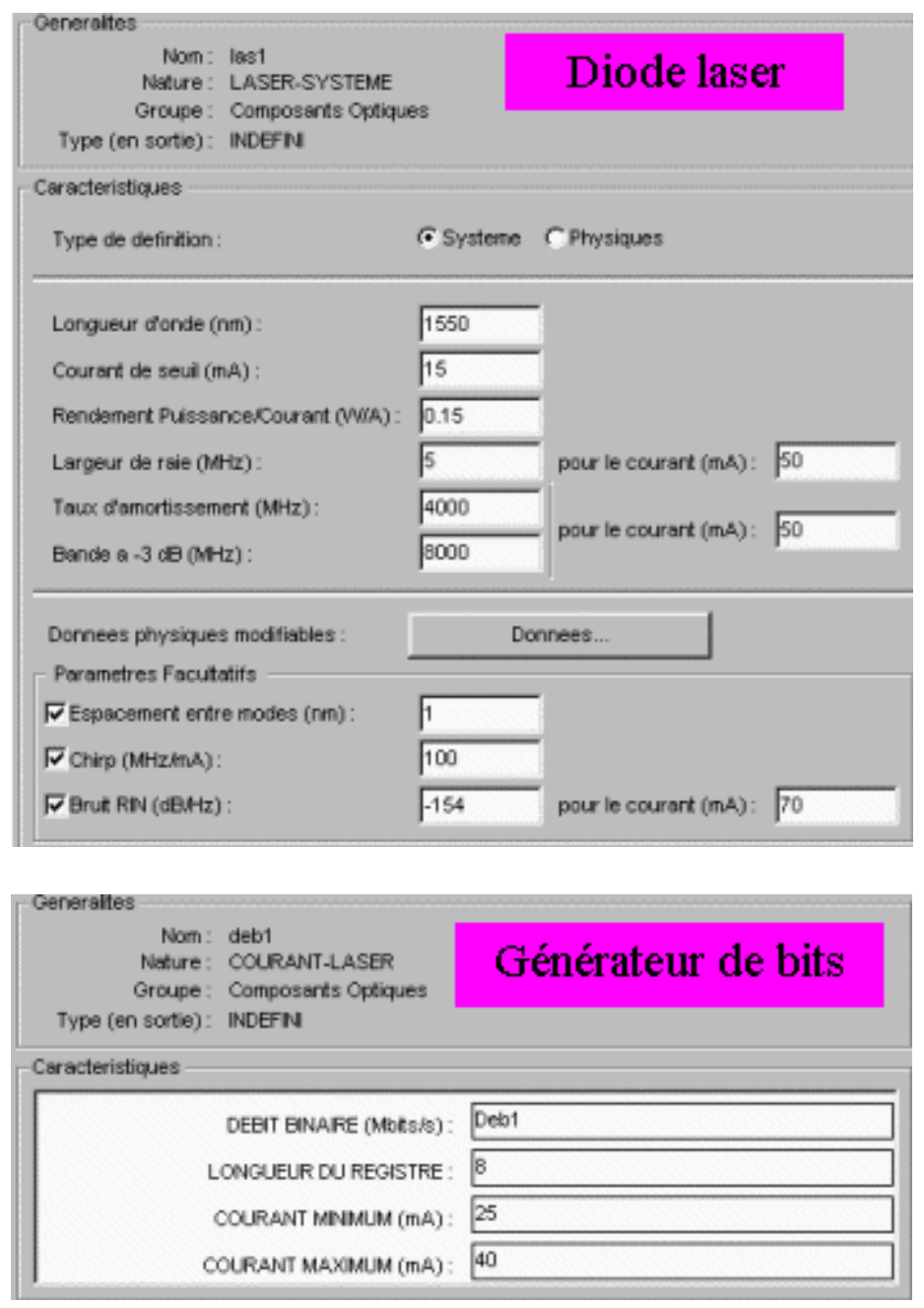


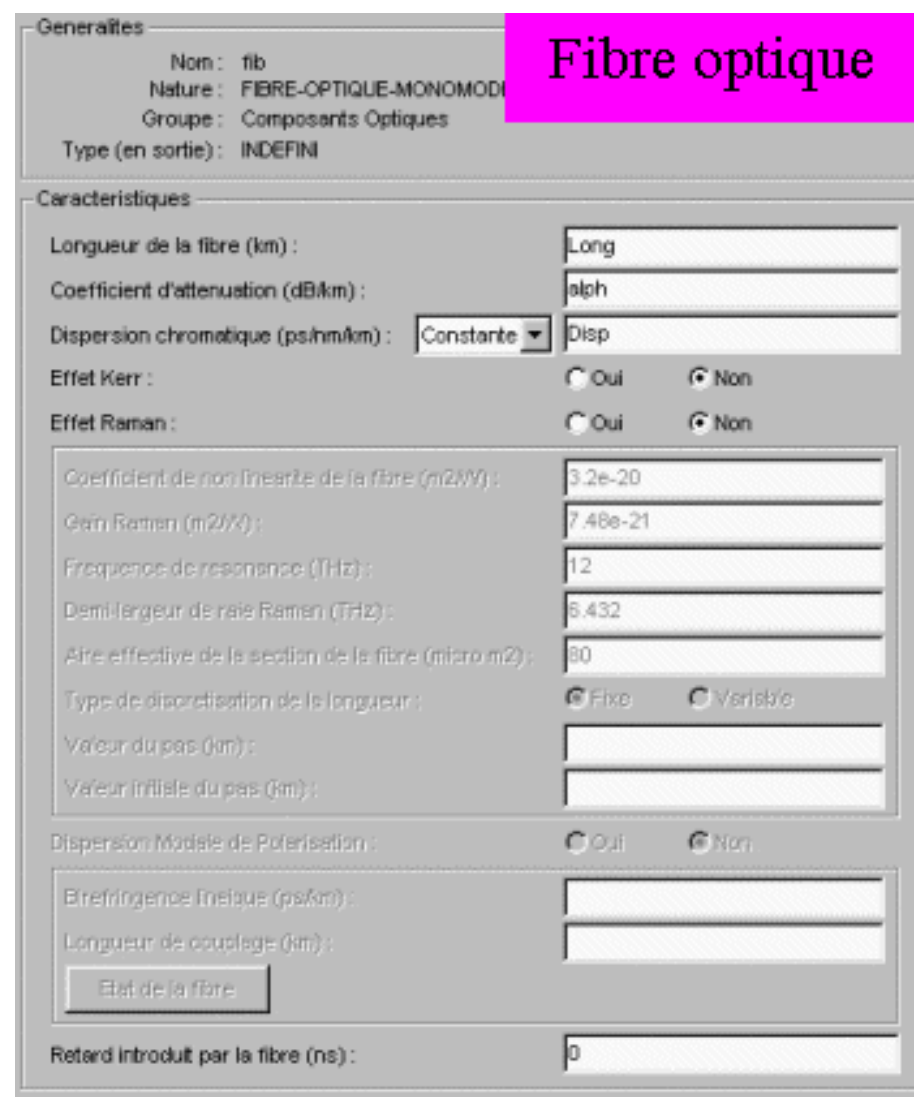

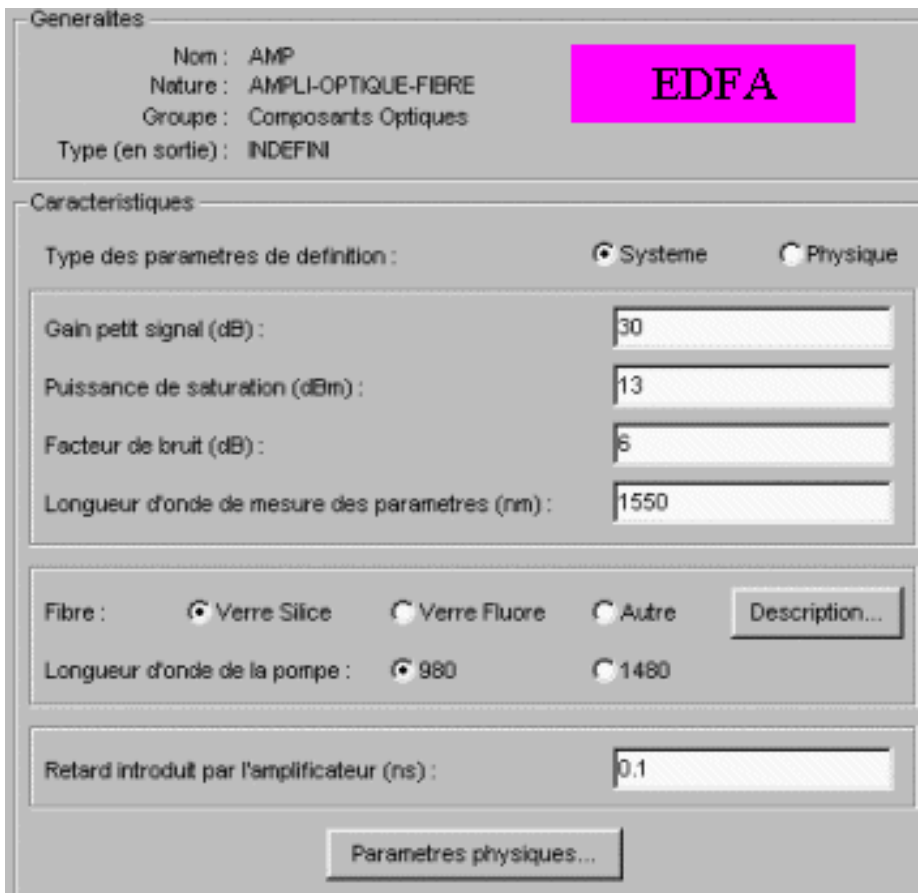

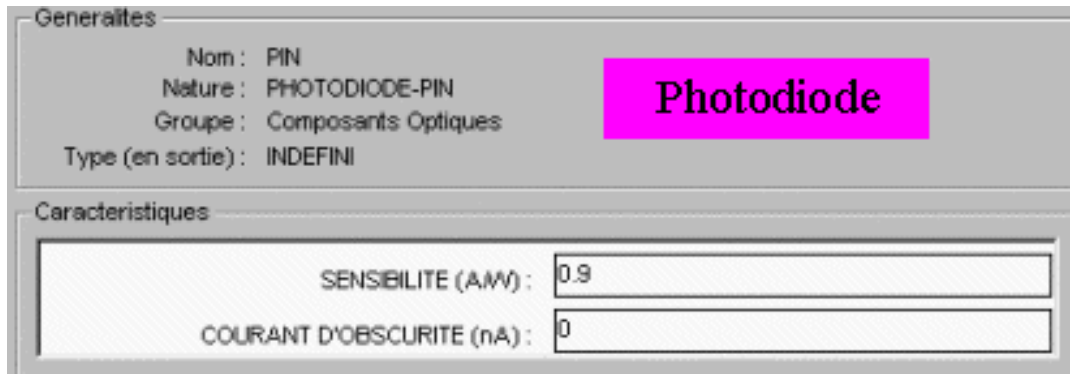


Fig.H1-1. Caractéristiques des éléments de la liaison point à point

Source : Copie d'écran du logiciel COMSIS [1] (voir également la figure 2).

\section{H.2. Caractéristiques statiques de l'EDFA : exemple à partir du logiciel COMSIS (EXP 1)}

La description de l'EDFA fournie au logiciel comporte les éléments suivants :

- $\quad$ gain $G=30 \mathrm{~dB}$

- $\quad$ facteur de bruit $F=6 \mathrm{~dB}$

- $\quad$ longueur d'onde centrale $\lambda=1,55 \mu \mathrm{m}$

La figure H2-1 montre la courbe de gain de l'amplificateur optique, lorsque le signal à amplifier est de « faible » puissance

$$
P_{\text {nn }} \& P_{\text {saturation }} \approx \mathrm{mW}
$$

( $P^{\prime}$ ). Dans ce cas, le signal de pompe est peu « consommé », et l'inversion de population en ions $\mathrm{Er}^{3+}$ est maintenue à un taux élevé. Le gain optique obtenu autour de la longueur d'onde $\lambda=1,31 \mu \mathrm{m}$, dont on note qu'il n'est pas plat, est élevé (de l'ordre de $35 \mathrm{~dB}$ ).

La figure H2-2 montre l'effet de la puissance de pompe seule sur le gain de l'amplificateur (gain relatif au signal à amplifier). On note que si la puissance de pompe est insuffisante, l'inversion de population des ions $\mathrm{Er}^{3+}$ est inefficace, le gain de l'amplificateur tend vers zéro. En revanche, toutes choses étant égales par ailleurs, le gain sature lorsque la puissance de pompe devient suffisante pour ne plus être entièrement consommée par le signal à amplifier. L'axe des abscisses permet de chiffrer l'ordre de grandeur de la puissance de pompe nécessaire dans le cas présent $(>50 \mathrm{~mW})$.

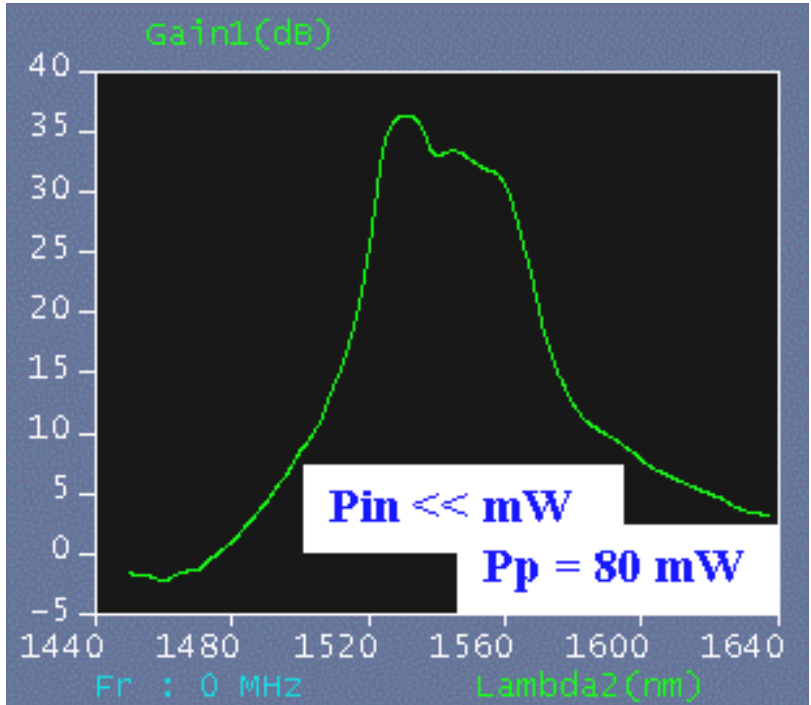

Fig. H2-1. Courbe de gain de l'amplificateur optique.

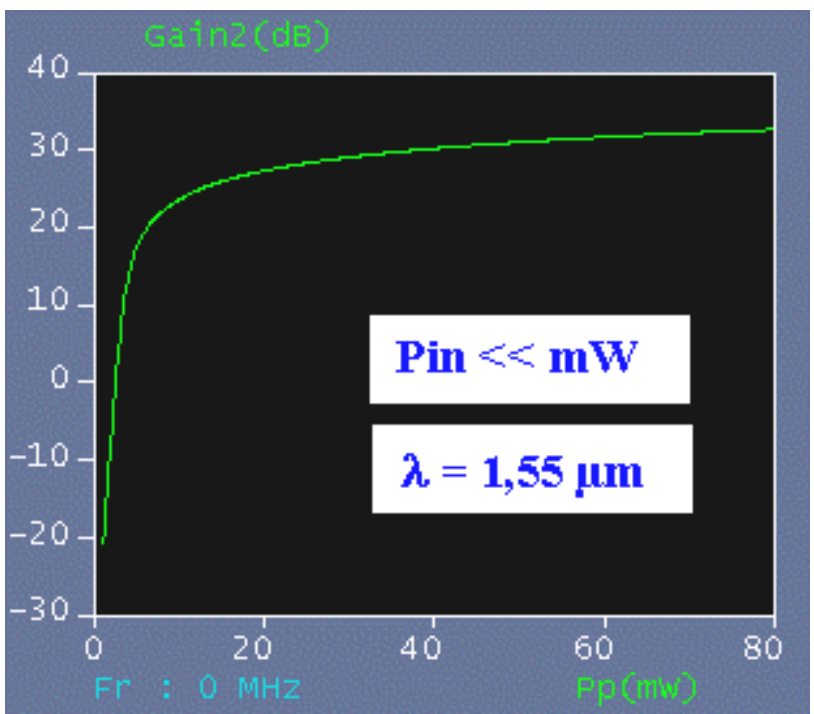

Fig. H2-2. Effet de la puissance de pompe seule sur le gain de l'amplificateur. 
La figure H2-3 montre l'effet de la puissance $\mathrm{P}_{\text {in }}$ du signal à amplifier sur le gain de l'amplificateur : toutes choses étant égales par ailleurs, en particulier la puissance de pompe (ici $80 \mathrm{~mW}$ ), le gain décroît lorsque la puissance $\mathrm{P}_{\text {in }}$ est trop forte. On parle de saturation du gain de l'amplificateur. $\mathrm{La}$ puissance de pompe permet en effet une inversion de population donnée des ions $\mathrm{Er}^{3+}$. Dans le même temps, l'émission stimulée de photon « signal » consomme l'inversion de population par passage d'ions $\mathrm{Er}^{3+}$ de l'état excité au niveau de base. Tant que le signal à amplifier est de faible puissance, l'inversion de population reste peu affectée. Lorsque celle-ci augmente, l'inversion de population en ions $\mathrm{Er}^{3+}$ diminue, donc le gain également. On chiffre là aussi le niveau de signal que l'amplificateur peut amplifier de manière satisfaisante.

La figure H2-4 présente la densité spectrale de puissance de bruit par amplification de l'émission spontanée (ASE, Amplification of Spontaneous Emission), qui est la principale source de bruit des amplificateurs EDFA. On note que la forme générale de la courbe est la même que celle du gain de l'amplificateur. Qualitativement, ceci s'explique par le fait que l'amplification optique conduit à une amplification du bruit (initialement blanc). La puissance de bruit a donc finalement la même forme de spectre optique. Compte tenu du débit de la liaison, il est possible de chiffrer l'ordre de grandeur de la puissance de bruit. Le niveau moyen étant de $-120 \mathrm{dBm} / \mathrm{Hz}$, soit $10^{15} \mathrm{~W} / \mathrm{Hz}$, si le débit est de 1 Gbits.s ${ }^{-1}$, la puissance d'ASE est de l'ordre de 1 microWatt. Il faut donc veiller à ce que le signal à amplifier ne sature pas l'amplificateur, mais ne tombe pas non plus en-dessous de ce niveau de puissance, car alors l'information risque d'être altérée.

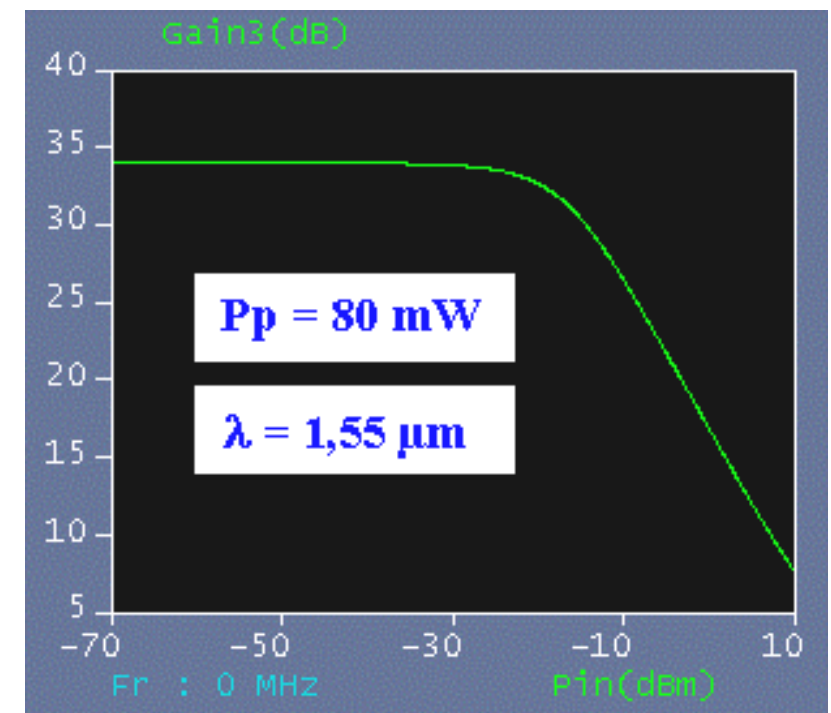

Fig. H2-3. Effet de la puissance de la puissance $\mathrm{P}_{\text {In }}$ sur le gain de l'amplificateur.

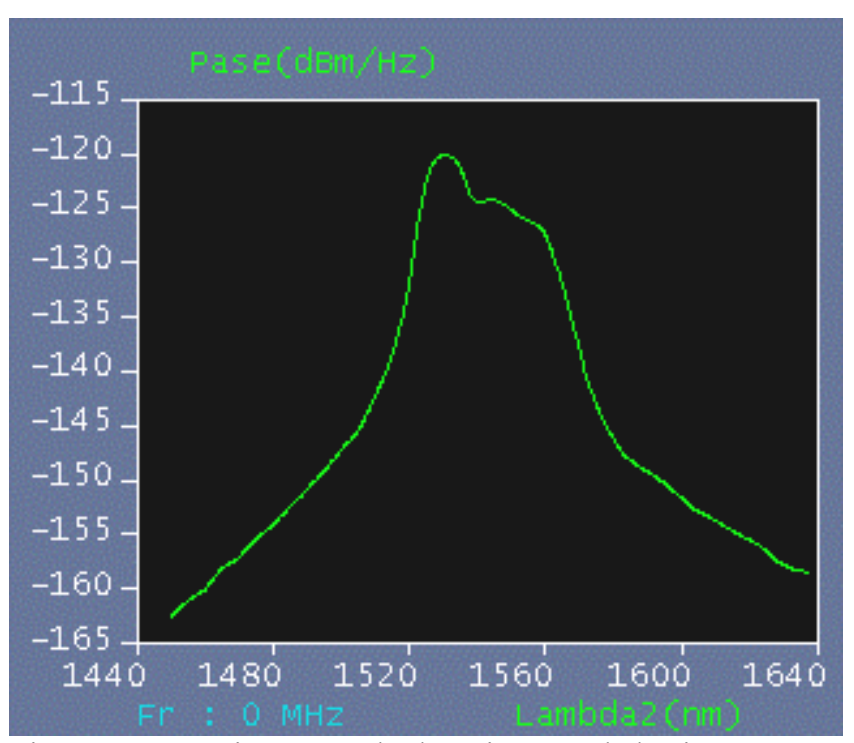

Fig. H2-4. Densité spectrale de puissance de bruit par amplification de l'émission spontanée (ASE).

Source : Copies d'écran du logiciel COMSIS [1] 
Annexe H.3. Effet du débit binaire sur la forme des impulsions délivrées par la diode laser (EXP 2)
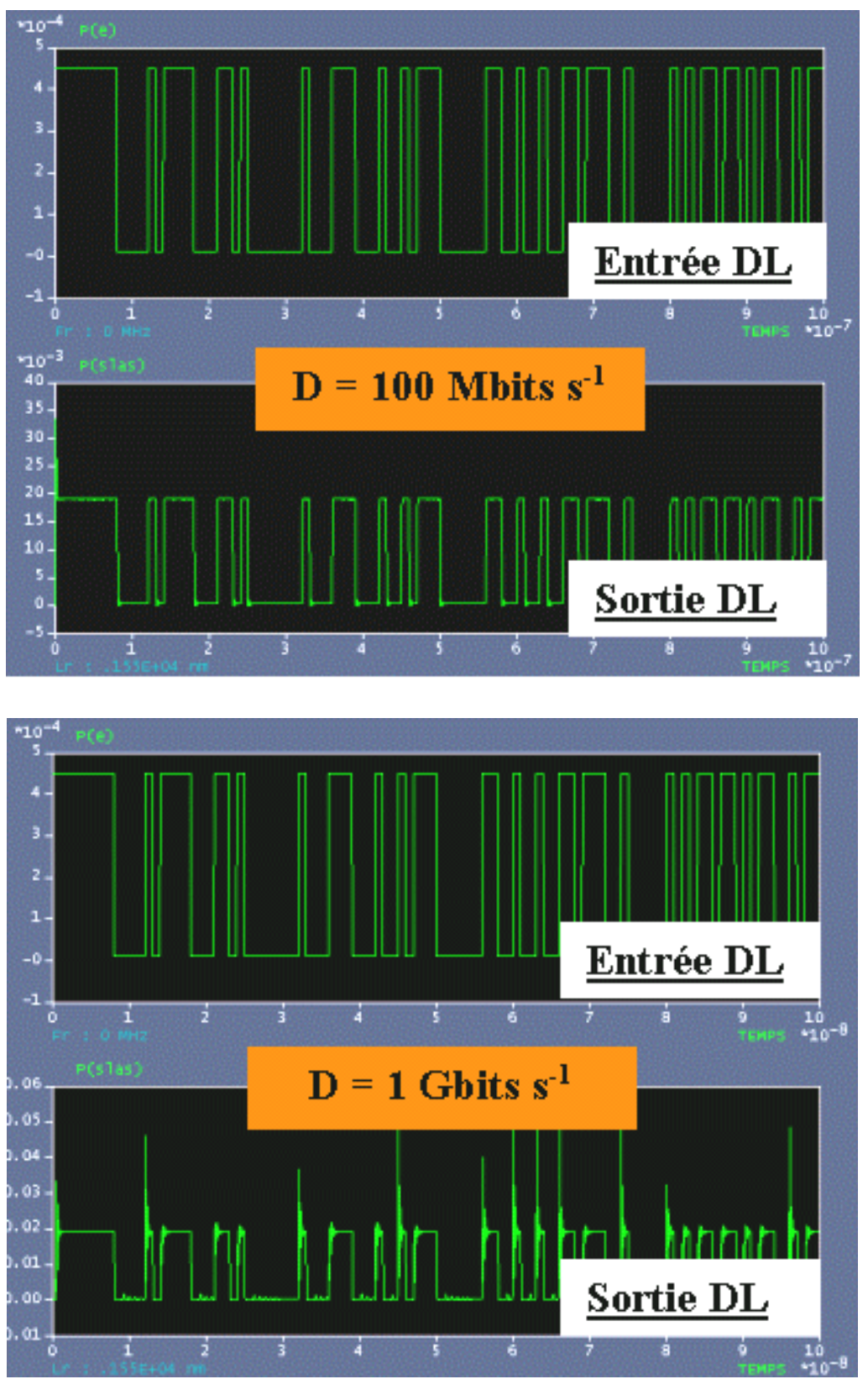


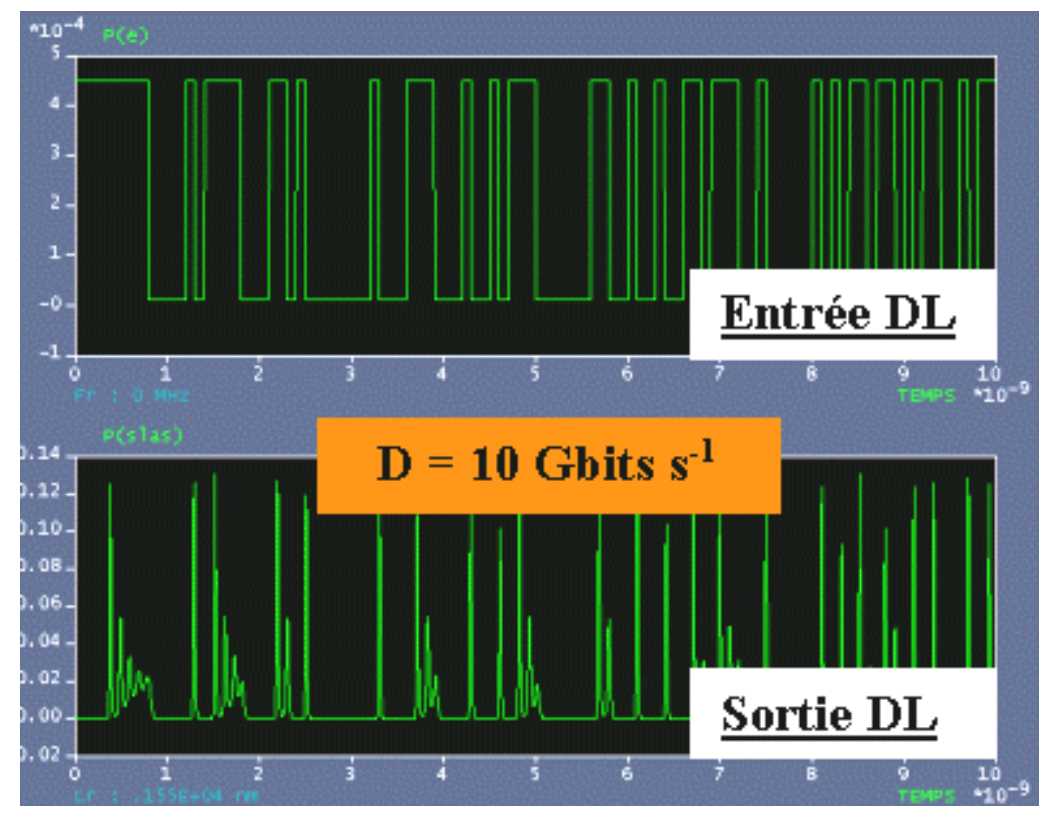

Fig. H3-1. Forme temporelle des impulsions de lumière délivrées par la diode laser. Source : Copies d'écran du logiciel COMSIS [1]

On note l'effet des oscillations de relaxation dès que le débit binaire dépasse $1 \mathrm{Gbits}^{-1} \mathrm{~s}^{-1}$ avec le modèle de diode laser considéré. Les impulsions sont alors loin d'être idéales. L'impact de la déformation engendrée à tel ou tel débit ne peut être évalué qu'en analysant globalement la liaison optique, par exemple au moyen d'un diagramme de l'oeil.

Annexe H.4. Dispersion chromatique seule de la fibre optique (EXP 3)

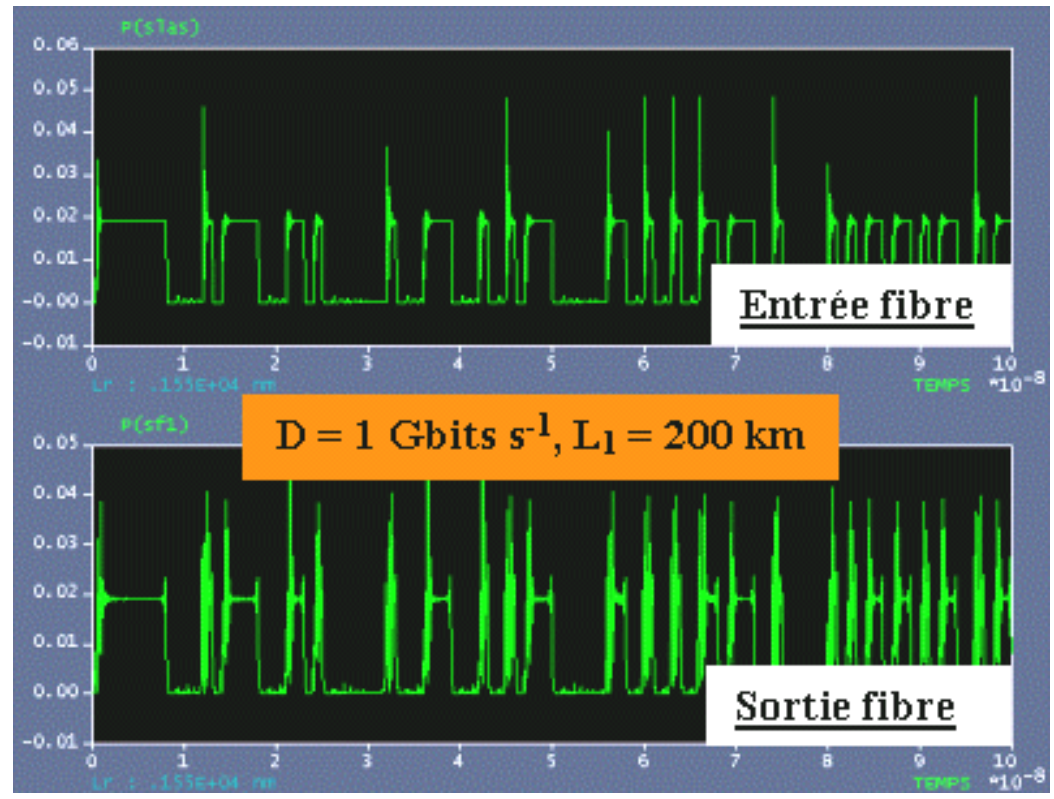



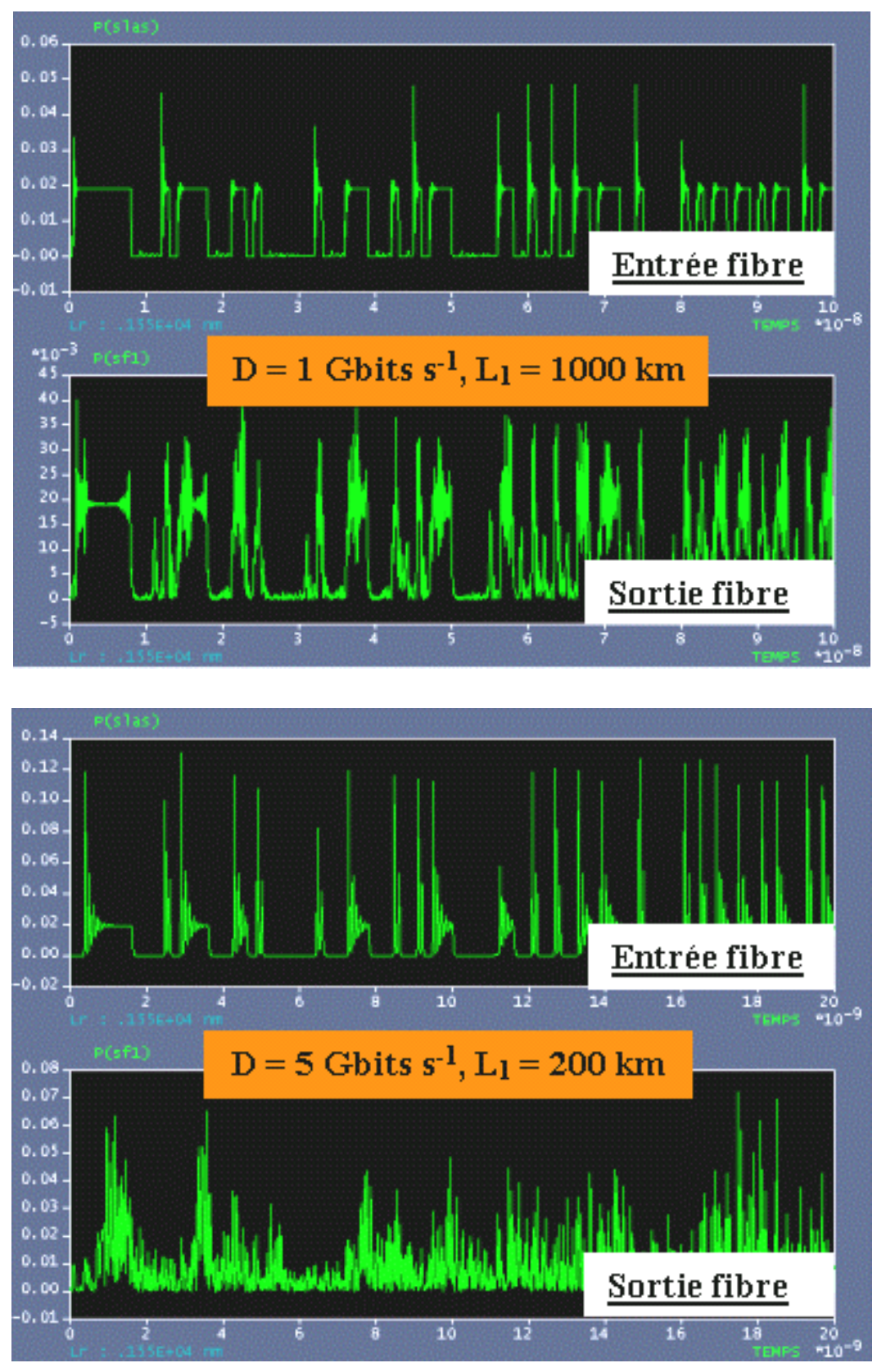

Fig. H4-1. Forme temporelle des impulsions de lumière après propagation dans la fibre optique. Source : Copies d'écran du logiciel COMSIS [1] 
Annexe H.5. Effet de la puissance du signal optique et de la puissance du signal de pompe de l'EDFA sur les signaux de la liaison point à point mono-longueur d'onde (EXP 4)

Dans ce cas, la puissance optique en entrée de l'EDFA est de l'ordre de $15 \mathrm{~mW}$ (pour le niveau haut), c'est-à-dire 11,8 dBm. Ce niveau de puissance est de l'ordre de la puissance de saturation de l'amplificateur (13 mW). Le gain de l'EDFA sature donc nettement. Du coup, le gain n'est que de 10 , soit $10 \mathrm{~dB}$. L'amplificateur ne fonctionne pas dans des conditions optimales.

Dans ce cas, la puissance optique en entrée de l'EDFA est de l'ordre de $70 \mu \mathrm{W}$ (pour le niveau haut), c'est-à-dire -11,5 dBm. Le gain de l'EFDA sature donc très peu : la puissance de sortie est de $90 \mathrm{~mW}$ environ, le gain est donc de $31 \mathrm{~dB}$ environ, ce qui est cohérent avec la caractéristique statique de l'EDFA. L'amplificateur optique fonctionne dans des conditions pratiquement idéales.
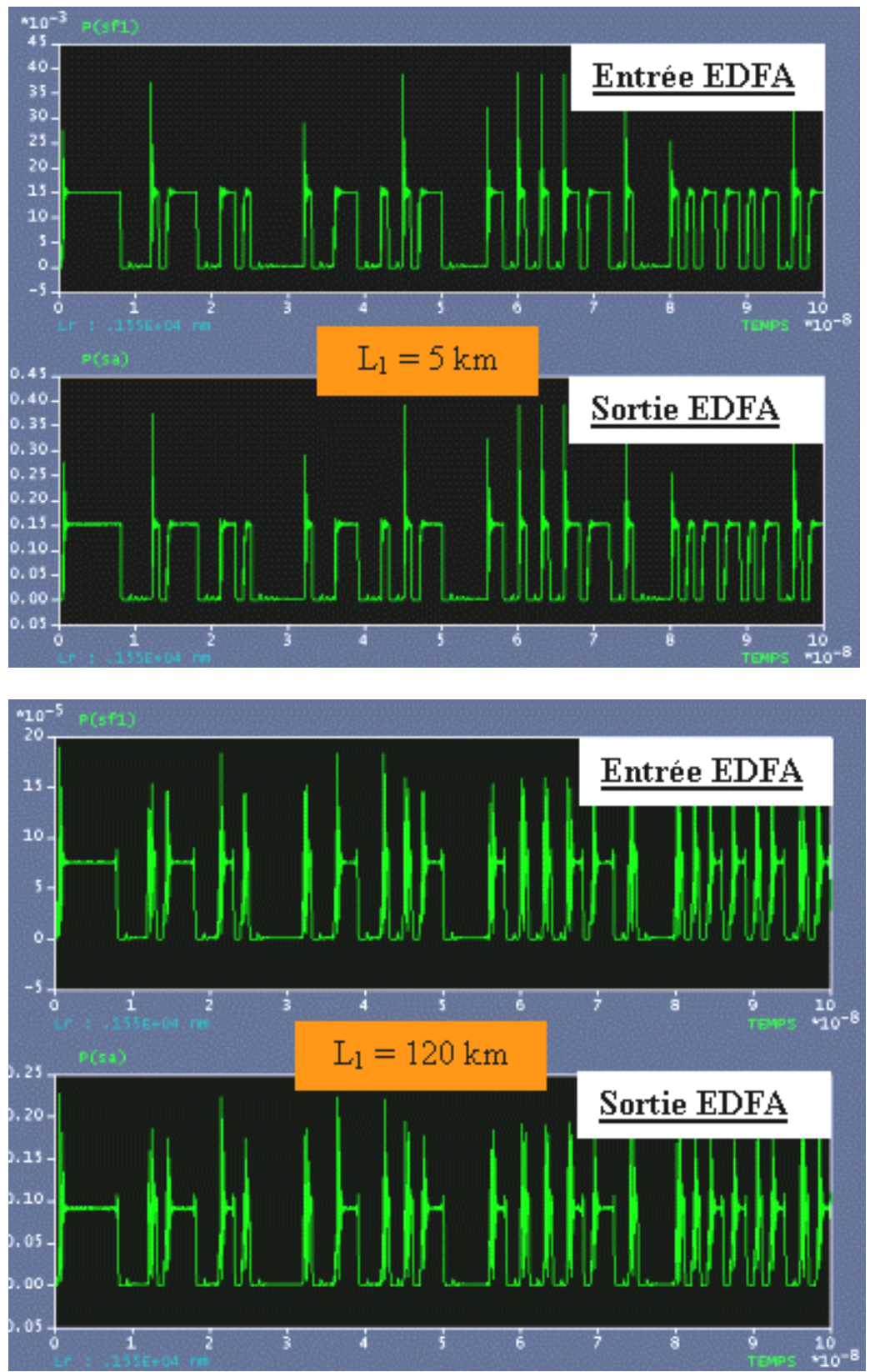
Dans ce cas, la puissance optique en entrée de l'EDFA est de l'ordre de $20 \mathrm{nW}$ (pour le niveau haut), c'est-à-dire $-47 \mathrm{dBm}$. Au regard des caractéristiques de gain de l'amplificateur optique, cette situation peut sembler favorable car le gain de l'EDFA ne sature pas du tout. En revanche, le niveau de puissance du signal est si faible qu'on amplifie davantage l'émission spontanée (ASE) que les photons signal. Le niveau de puissance en sortie est effectivement relevé (gain de l'ordre de $35 \& n b s p d B$ ), mais la forme des impulsions est très altérée.

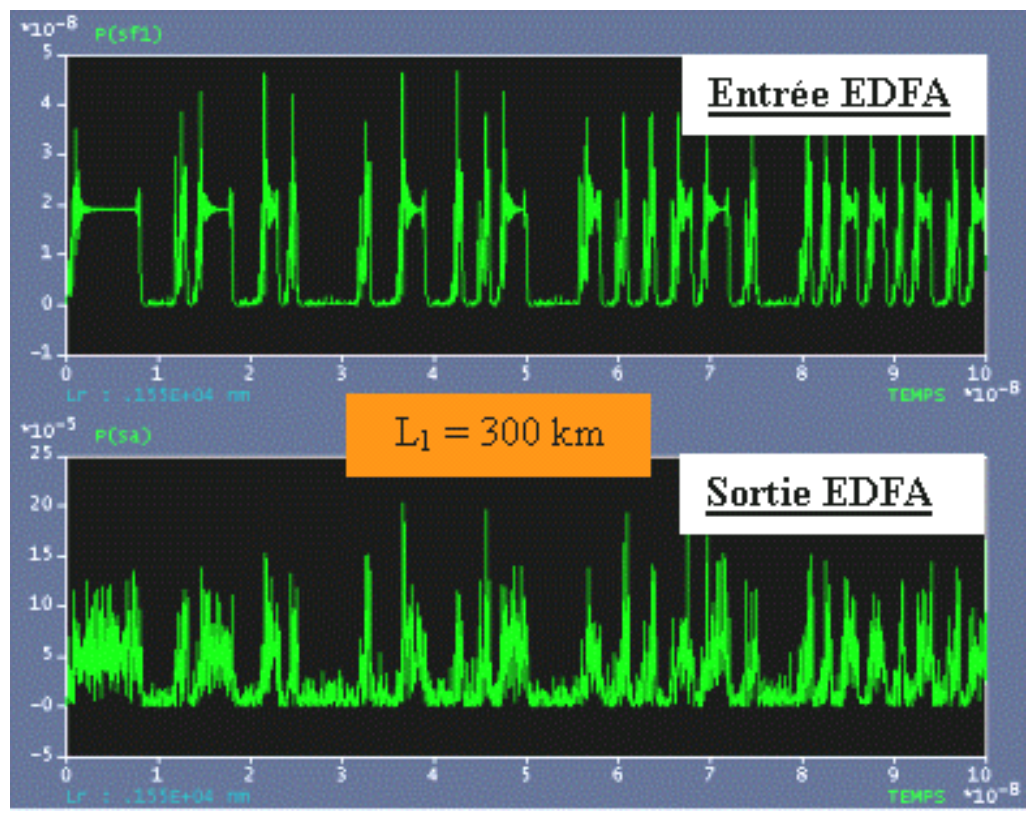

Fig. H5-1. Forme temporelle des signaux optiques en entrée et en sortie de l'EDFA.

\section{Annexe H.6. TEB et facteur Q}

Les notions de taux d'erreur par bit (TEB) et de facteur Q ne sont pas particulières aux liaisons par fibre optique, mais sont générales aux communications numériques.

Le TEB correspond à la probabilité d'erreur lors de la transmission d'un bit d'information. Compte tenu des débits binaires utilisés usuellement dans les communications par fibre (environ $10 \mathrm{Gbits}^{-1} \mathrm{~s}^{-1}$ ), un TEB de $10^{-7}$ par exemple correspond à environ 1000 erreurs par seconde, ce qui est catastrophique ! Dès lors, on comprend que les TEB requis par les applications des télécommunications soient extrêmement faibles : il faut typiquement $\mathrm{TEB}<10^{-15}$.

En présence de sources de bruit (bruit thermique, bruit de grenaille, bruit optique dans les amplificateurs optiques, ...), les signaux reçus ne correspondent pas idéalement aux niveaux logiques « $0 »$ ou « $1 »$. C'est ce qu'indique la figure ci-contre, où $I_{0}$ et $I_{1}$ correspondent aux niveaux des signaux associés aux deux états logiques. Les signaux reçus en détection sont plutôt caractérisés par des densités de probabilité, centrées sur $I_{0}$ et $\mathrm{I}_{1}$. Dans la mesure où ces lois de probabilité sont gaussiennes, $\sigma_{0}^{2}$ et $\sigma_{1}^{2}$ représentant les variances de bruit en courant des deux états, on voit que la probabilité d'erreur inter-symbôles, liée au recouvrement des densités de probabilité, n'est pas nulle. Il s'agit donc de choisir la meilleure valeur du seuil de décision $I_{T h}$ pour la prise de décision. On montre que pour la meilleure valeur de $I_{T h}$, TEB est donné par l'expression suivante :

$$
\mathrm{TEB}=\frac{1}{2} \operatorname{Erc}\left(\frac{Q}{\sqrt{2}}\right) \approx \frac{\exp \left(-\frac{Q^{2}}{\sqrt{2}}\right)}{Q \sqrt{2} \pi}(\text { si } Q>3) \text {, avec } Q=\frac{I_{1}-I_{0}}{\sigma_{0}+\sigma_{1}}
$$

En pratique, l'évaluation du facteur $Q$, à partir des différentes sources de bruit du circuit de détection, est effectuée dans un premier temps. Le TEB s'en déduit alors très simplement. 


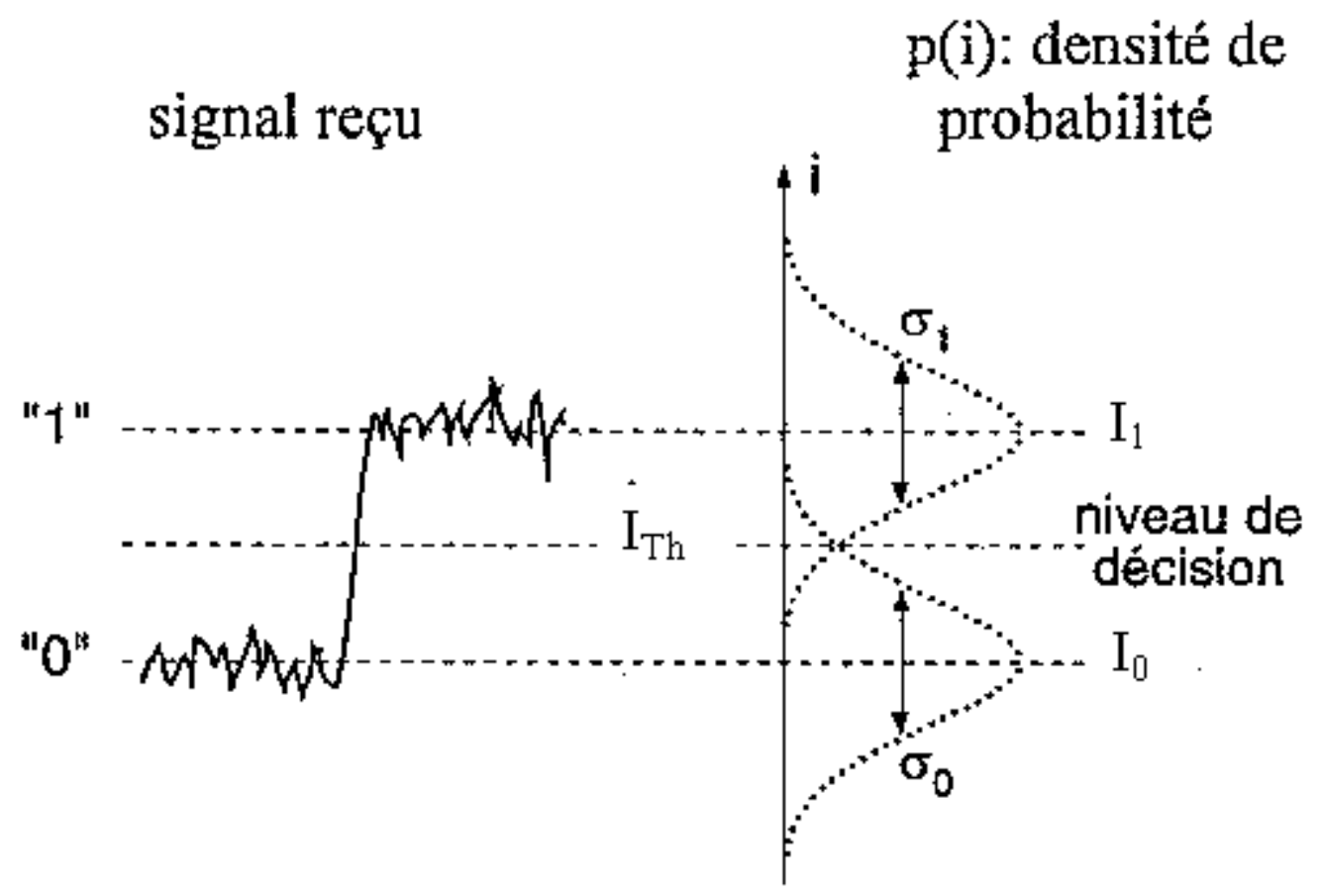

Fig. H6-1. Photocourants correspondant aux niveaux logiques « 0 » et « 1 » et densitéde probabilités associées.

\section{Annexe H.7. Diagramme de l'oeil de la liaison optique point à point}

- L'ouverture verticale renseigne sur la différence qui existe entre les niveaux logiques « $0 »$ et « $1 »$. Plus l'oeil est ouvert et plus la situation est favorable pour la discrimination des bits hauts et bas.

- L'ouverture horizontale renseigne sur le degré de « gigue » présent dans la liaison (toutes les communications synchrones sont très sensibles à ce défaut). Là il est souhaitable que l'ouverture de l'oeil soit la plus grande possible.

- L'épaisseur des lignes au niveau de l'axe horizontal donne également une indication sur la présence de " gigue ».

- En dernier lieu, c'est tout simplement l'ouverture de l'oeil elle-même qui renseigne sur la qualité de la liaison numérique, et dnc sur la facilité qu'il y aura à interpréter le flux de bits reçu. 


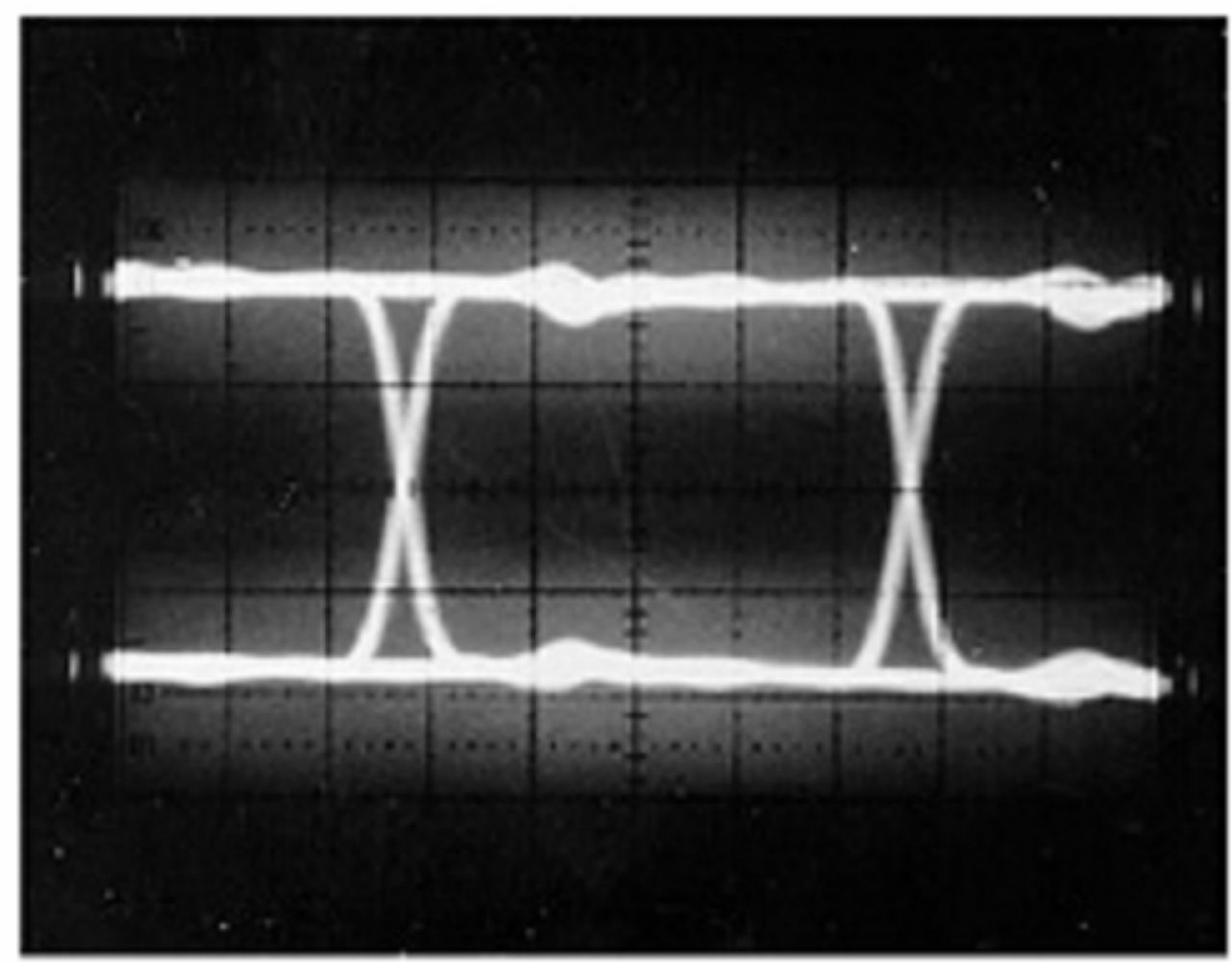

Fig. H7-1. Diagramme de l'oeil réel (dans un cas favorable).

\section{Annexe H.8. Diagramme de l'oeil de la liaison optique point à point (EXP 5)}

Configuration choisie : $L_{1}=L_{2}=50 \mathrm{~km}, \alpha=0,2 \mathrm{~dB} / \mathrm{km}, D_{\lambda}=17 \mathrm{ps} /(\mathrm{nm} . \mathrm{km})$

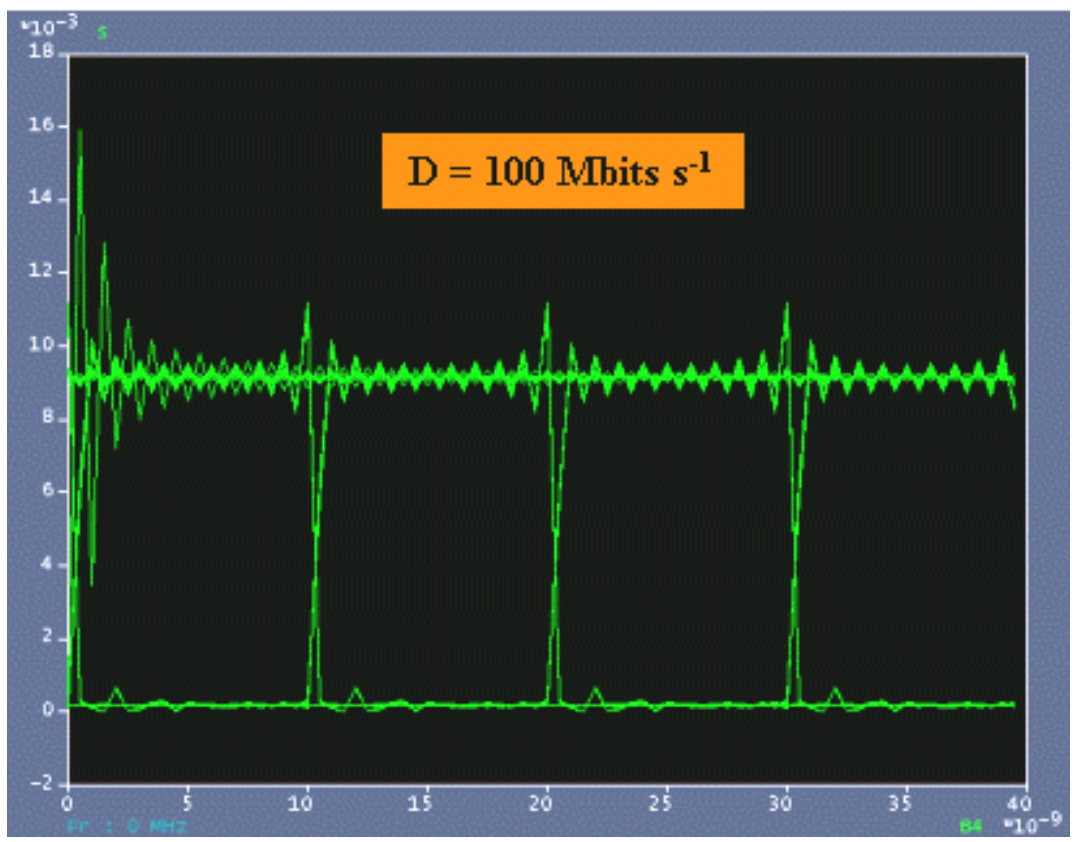




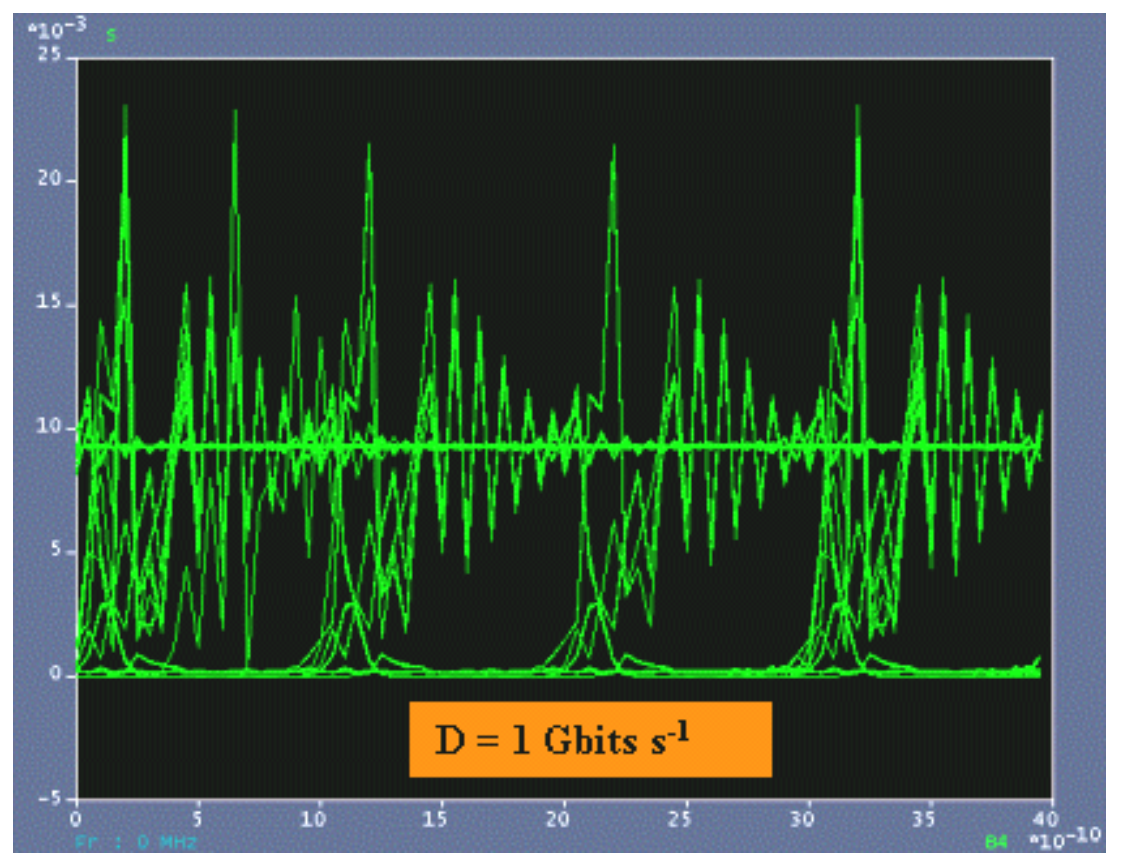

Fig. H8-1. Diagramme de l'oeil de la liaison optique point à point. Source : Copies d'écran du logiciel COMSIS [1]

Dans la configuration choisie, l'oeil présente une ouverture satisfaisante à $100 \mathrm{Mbits} . \mathrm{s}^{-1}$, mais il est déjà trop fermé à 1 Gbits.s ${ }^{-1}$.

\section{Annexe I. Schéma de la liaison WDM étudiée}

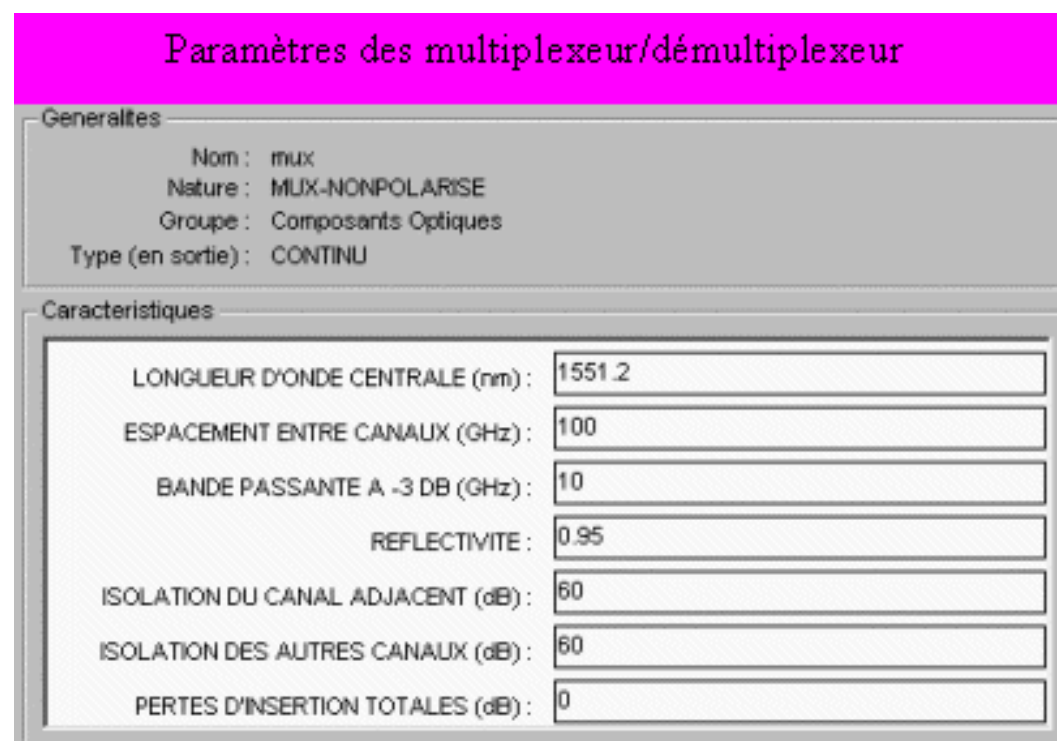

Fig. I-1. Paramètres des MUX/DEMUX en longueurs d'onde. 


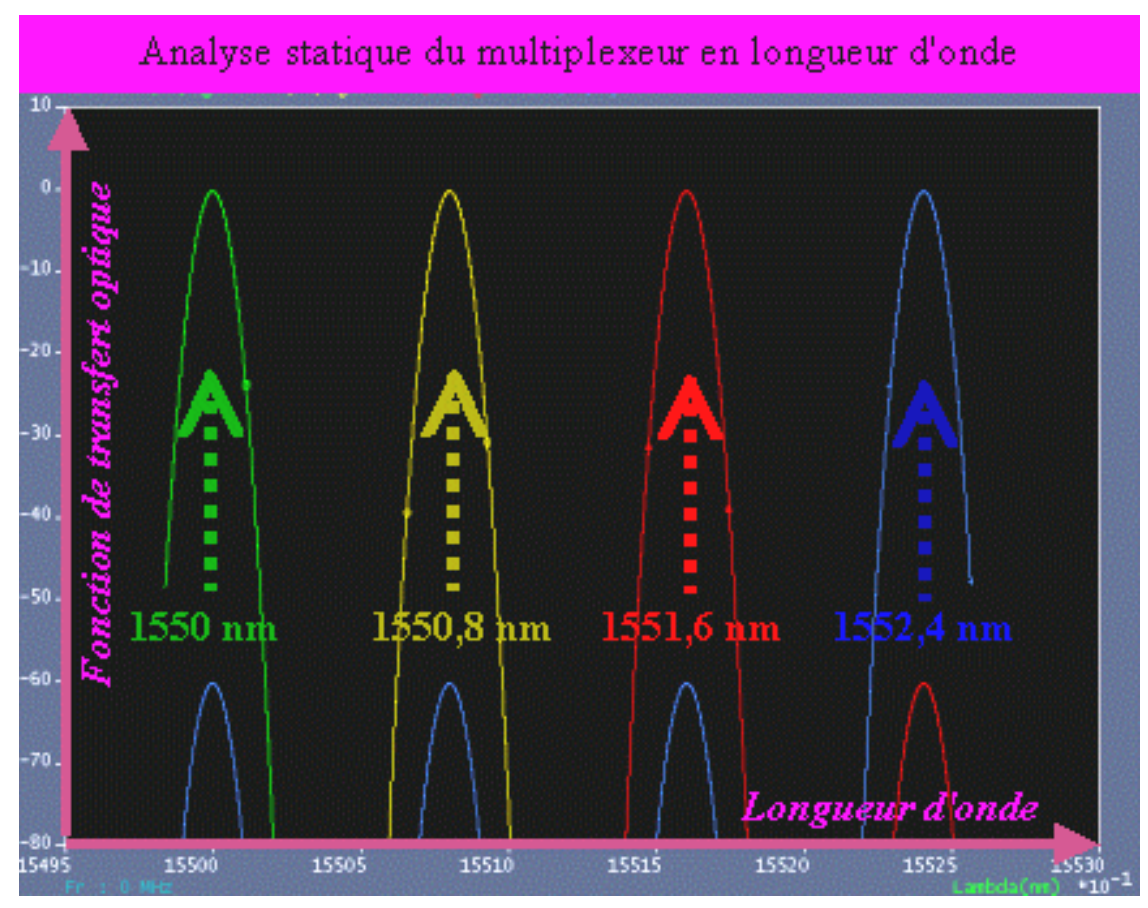

Fig. I-2. Caractéristique en longueur d'onde des MUX/DEMUX.

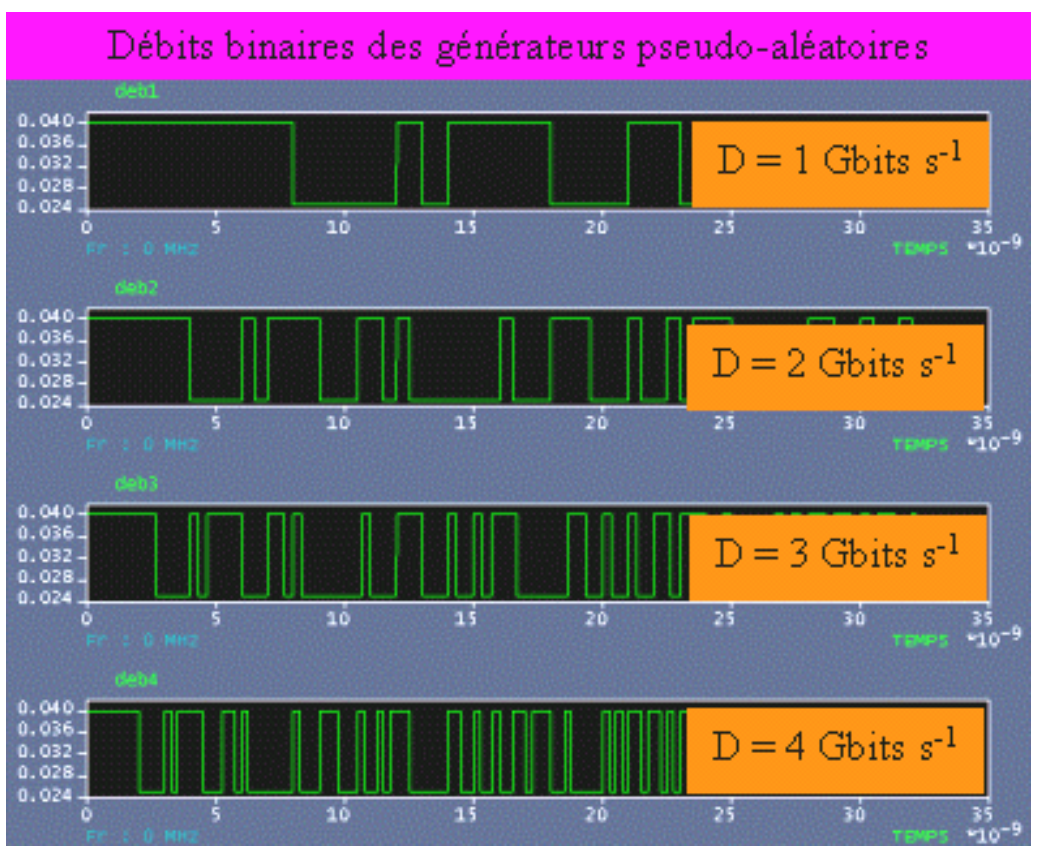

Fig. I-3. Forme temporelle des signaux optiques en sortie des généateurs pseudo-aléatoires. 


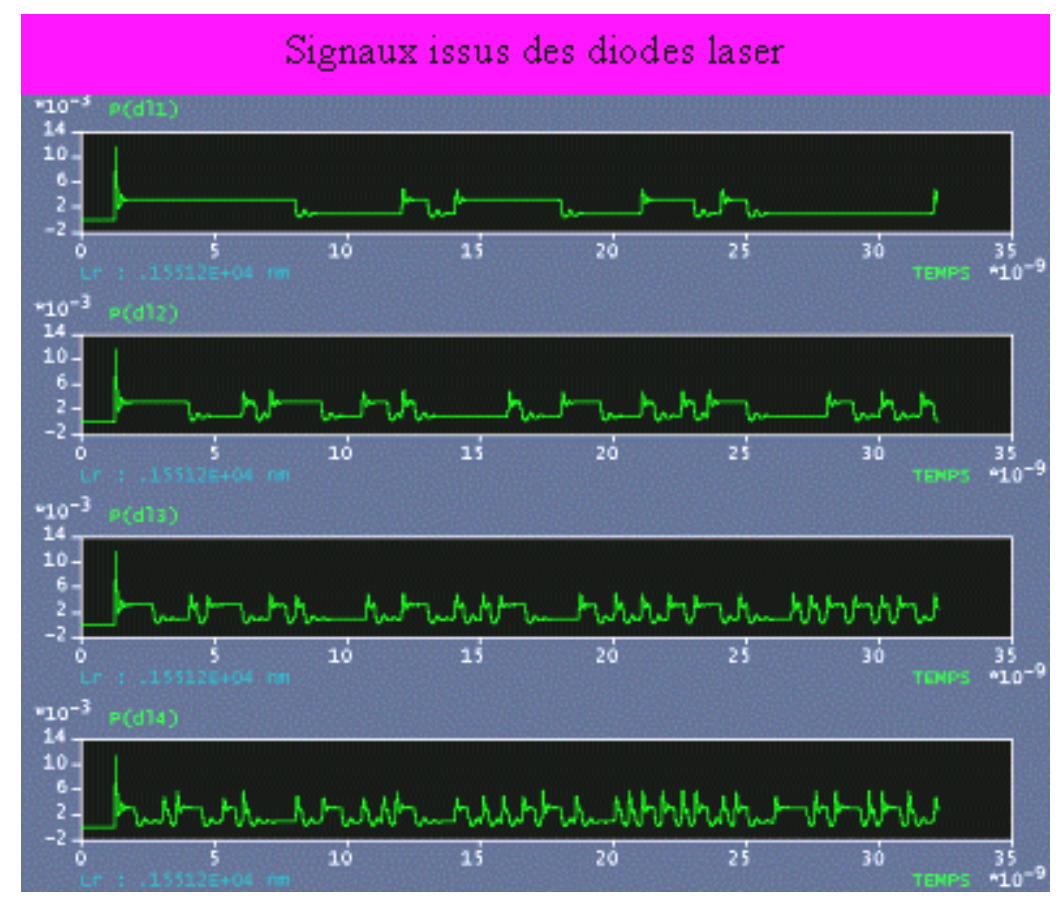

Fig. I-4a. Forme temporelle des signaux optiques en sortie des diodes laser.

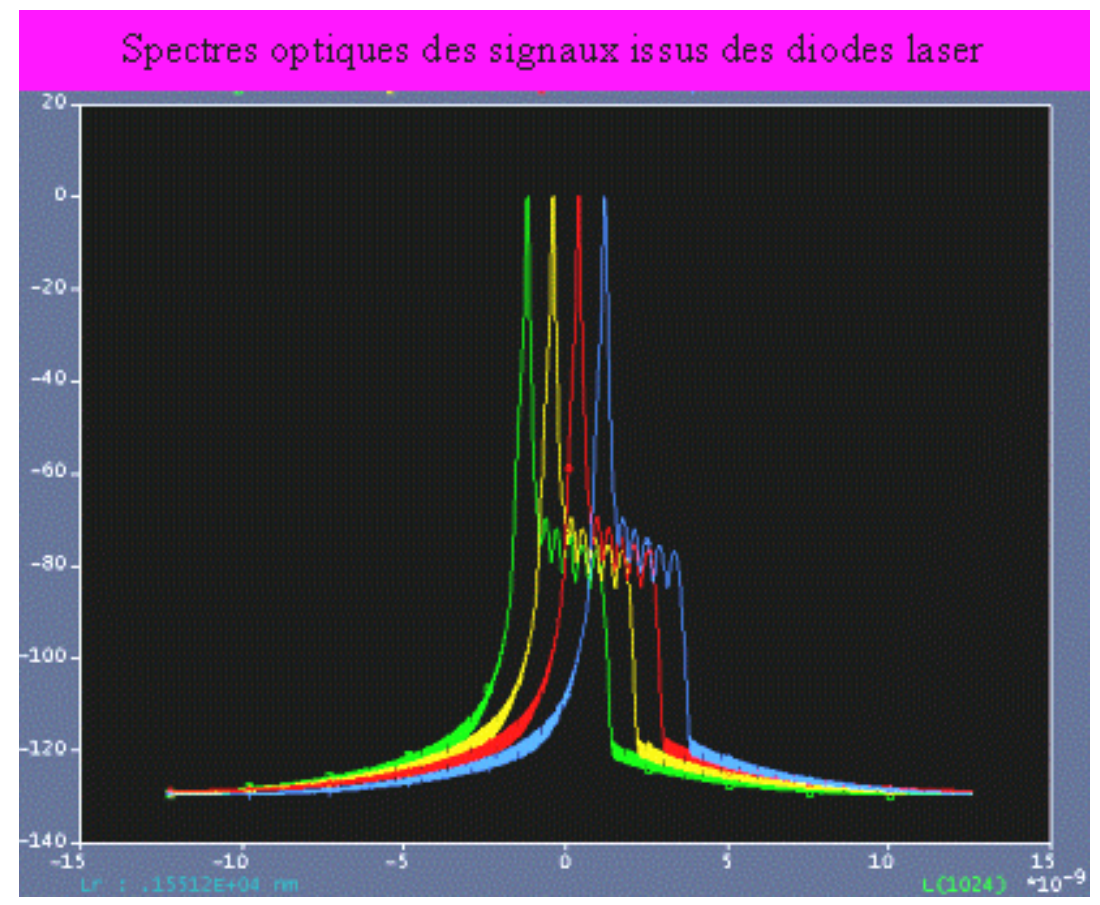

Fig. I-4b. Densité spectrale de puissance des signaux optiques en sortie des diodes laser. 


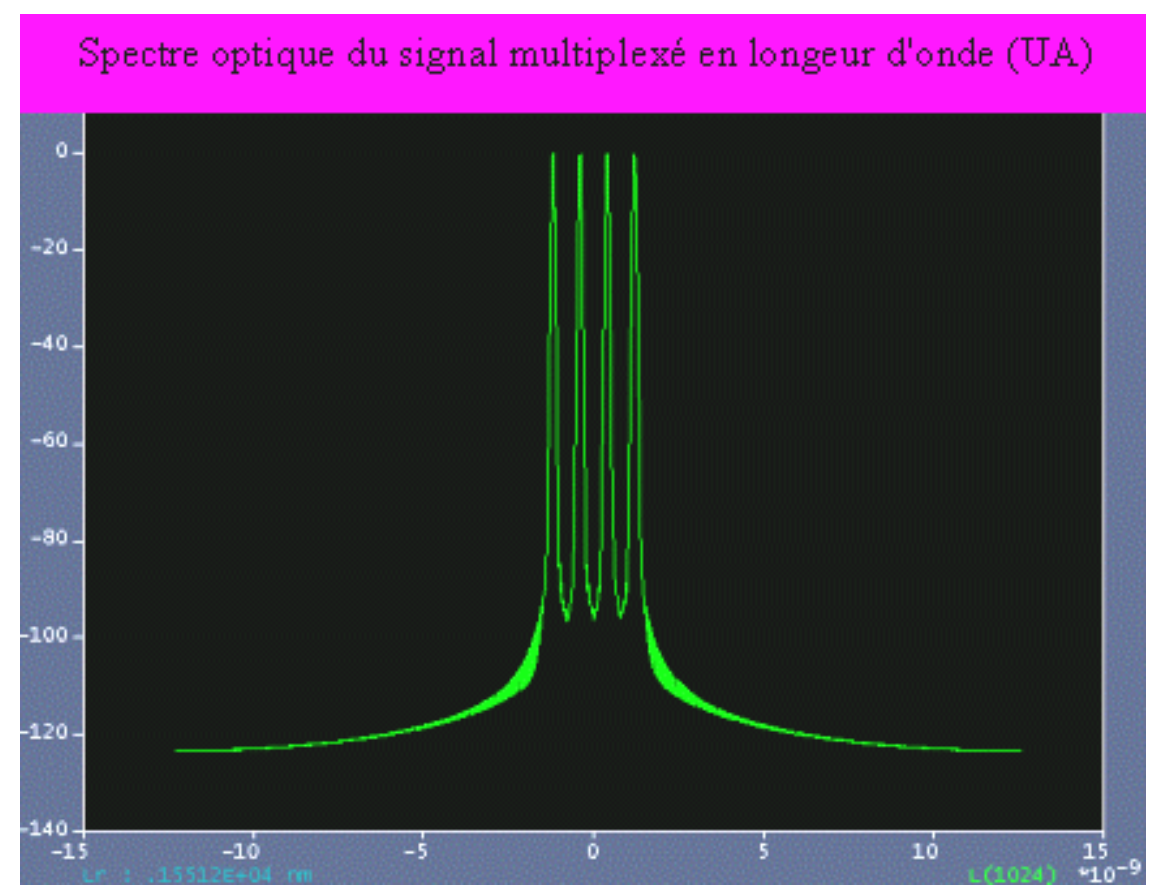

Fig. I-5a. Densité spectrale de puissance du signal optique en sortie de multiplexeur.

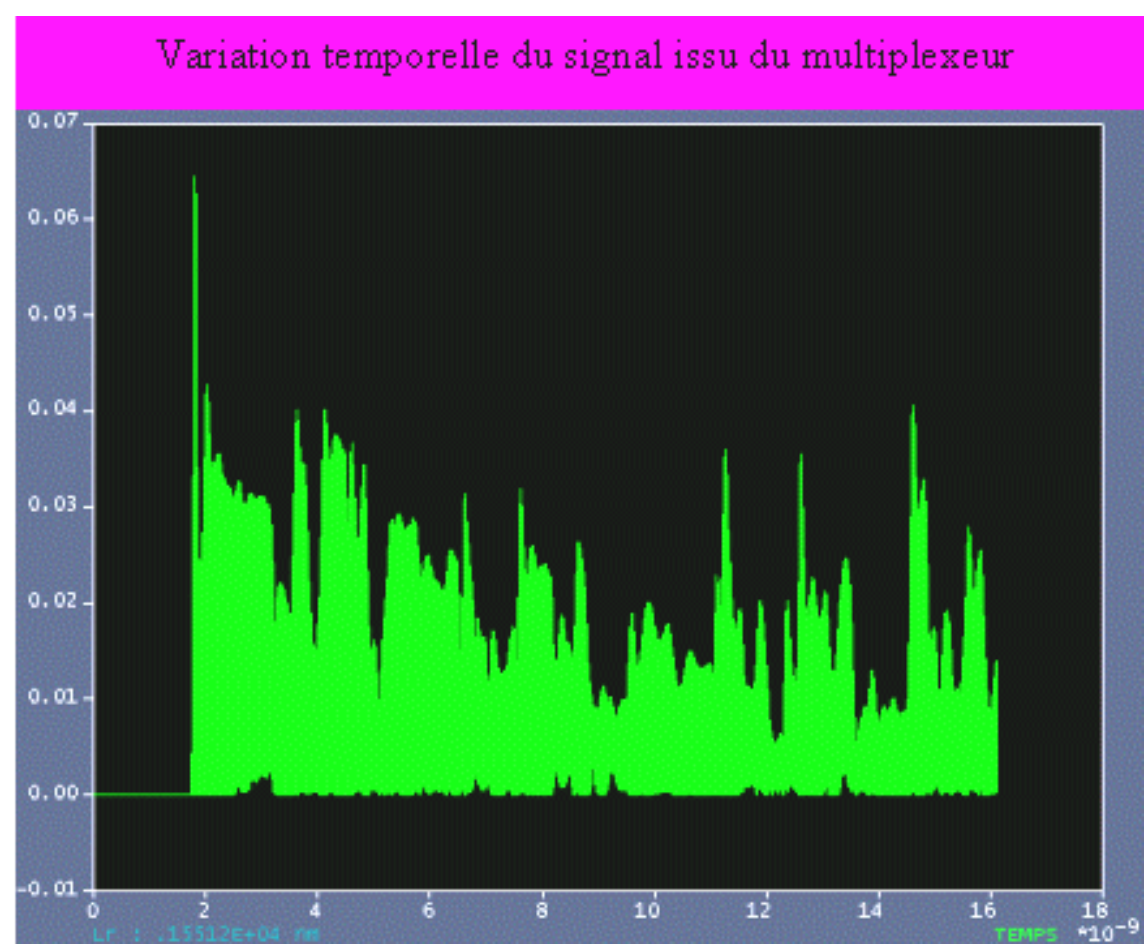

Fig. I-5b. Forme temporelle du signal optique en sortie de multiplexeur. 


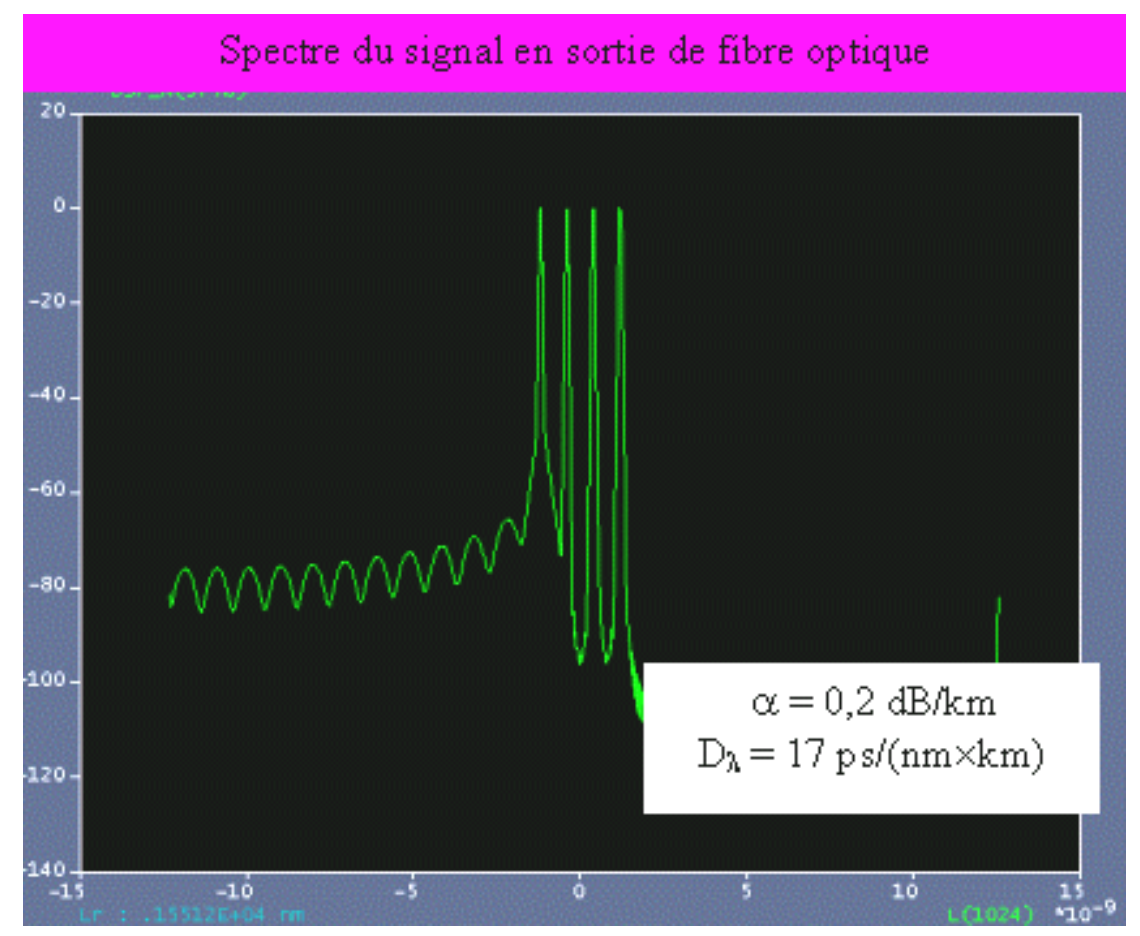

Fig. I-6a. Densité spectrale de puissance du signal optique en sortie de la section de fibre.

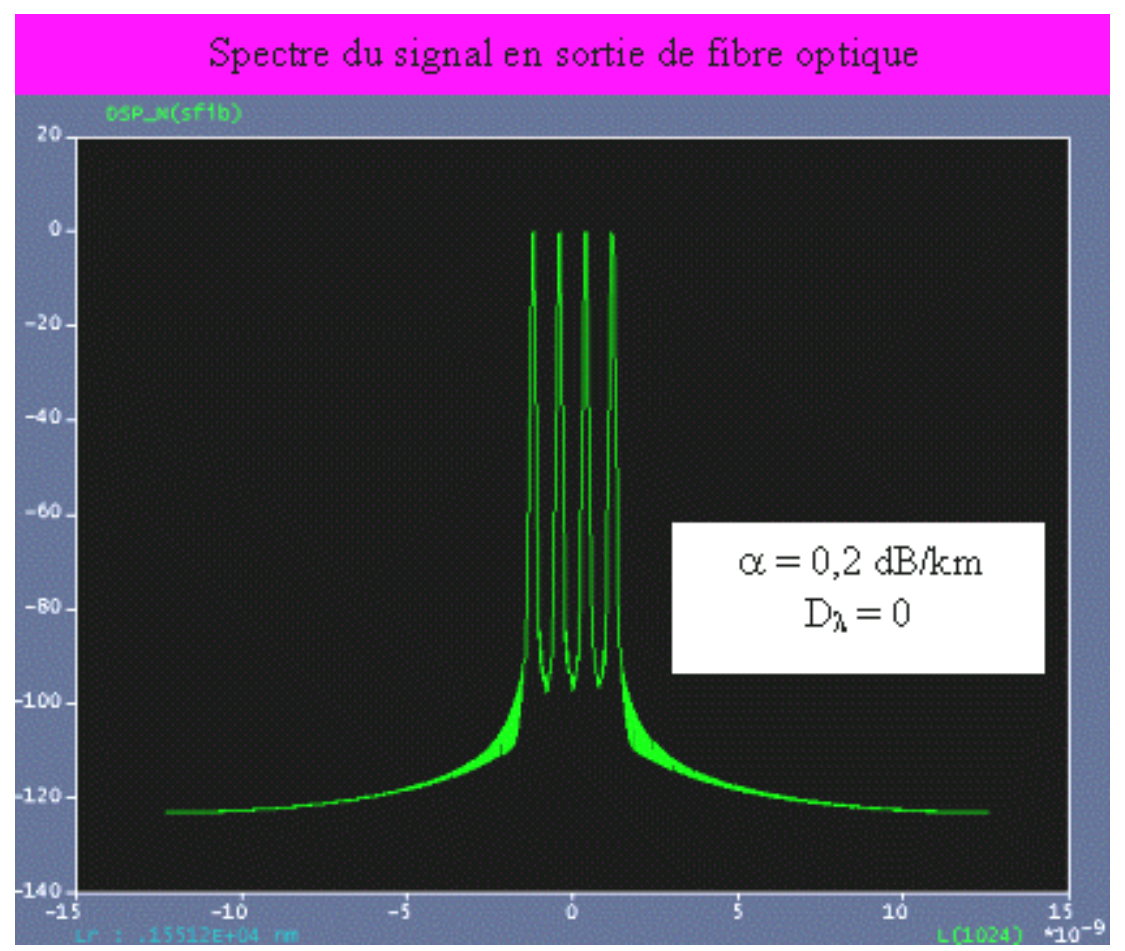

Fig. I-6b. Densité spectrale de puissance du signal optique en sortie d'une même section de fibre non-dispersive. 


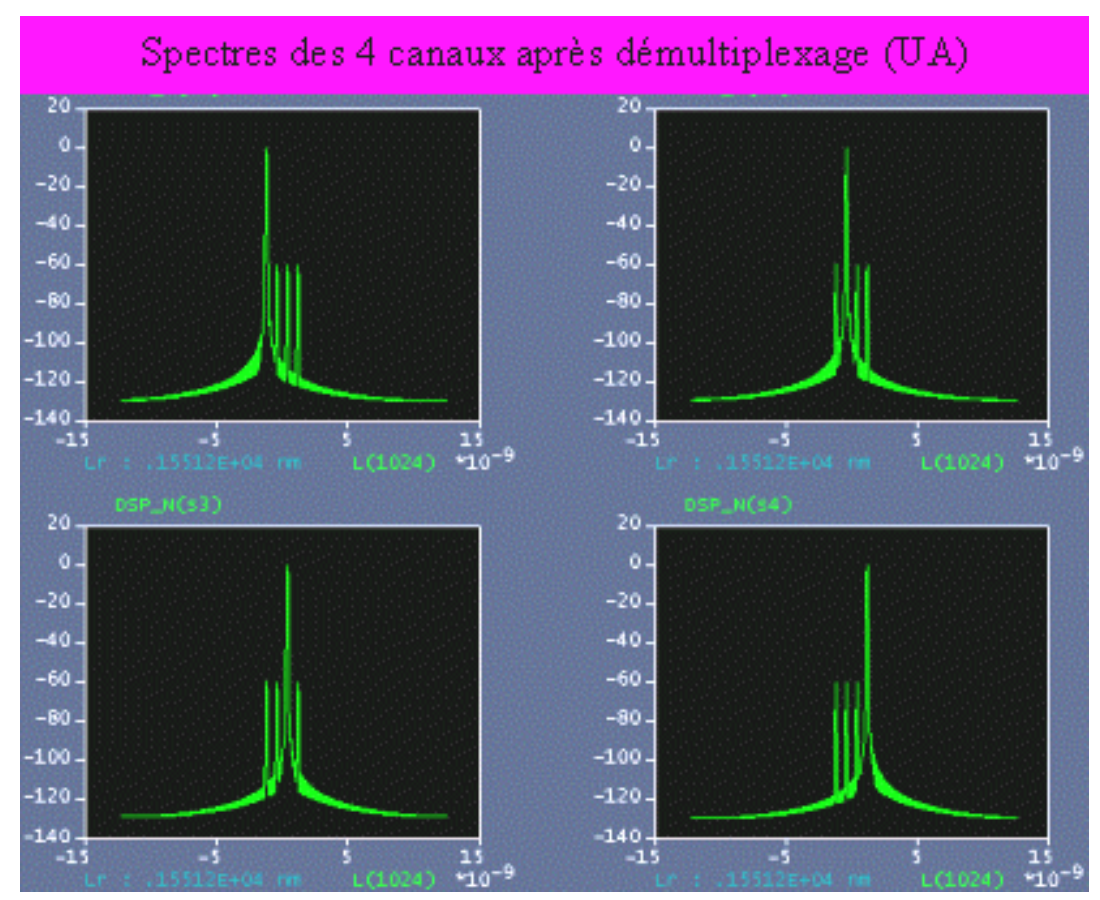

Fig. I-7a. Densité spectrale de puissance des signaux optiques en sortie dedémultiplexeur (signaux séparés).

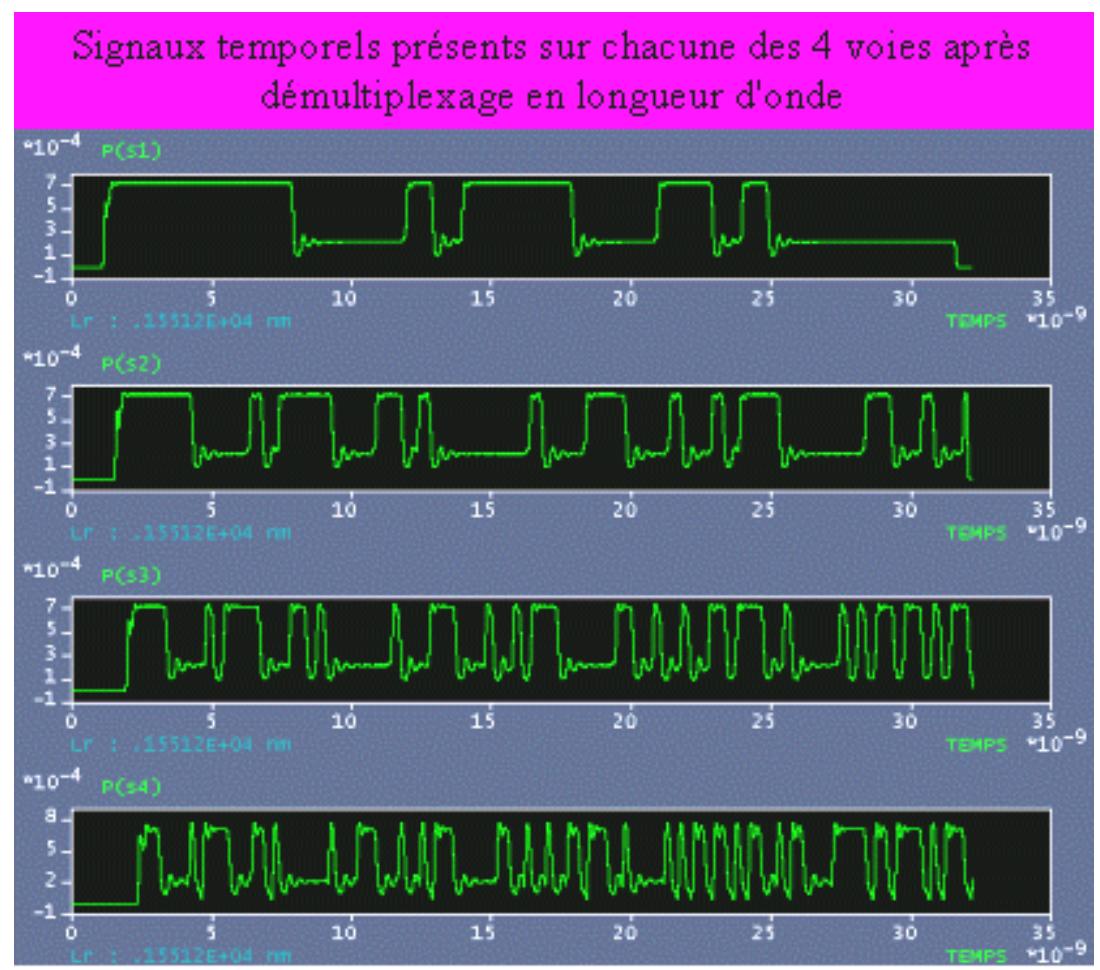

Fig. I-7b. Forme temporelle des signaux optiques en sortie de démultiplexeur (signaux séparés).

Source : Copies d'écran du logiciel COMSIS [1] 